COMMUNICATIONS IN

ANALYSIS AND GEOMETRY

Volume 11, Number 2, 343-389, 2003

\title{
Dehn surgeries, group actions and Seifert fiber spaces
}

\author{
Kiminiko Motegi $^{1}$
}

TABLE OF CONTENTS

1. Introduction

2. Outline of the paper

3. Isomorphisms of base orbifolds and detecting fibers

4. Seifert fibered surgeries on freely periodic knots

5. Strong inversions and Seifert fibered surgeries

6. Non-cyclic actions before/after Dehn surgeries

7. Reflections on base orbifolds

8. Determining knot types using finite group actions

9. Toroidal Seifert fibered surgeries for hyperbolic knots

10. Toroidal Seifert fibered surgeries for non-hyperbolic knots

11. Projective Seifert fibered surgeries on symmetric knots

12. Small Seifert fibered surgeries

\section{Introduction.}

Let $K$ be a knot in the 3 -sphere $S^{3}$ and $\gamma$ a slope (the isotopy class of an essential simple closed curve) on the boundary of the exterior of $K$. Then the manifold obtained from $S^{3}$ by $\gamma$-Dehn surgery on $K$ is denoted by $(K ; \gamma)$. As usual, using the preferred meridian-longitude pair of $K \subset S^{3}$, we parametrize slopes, and hence surgeries on $K$ by $r \in \mathbb{Q} \cup\{\infty\}$; we also write $(K ; r)$ for $(K ; \gamma)$. Suppose that $K$ is hyperbolic, i.e., the complement $S^{3}-K$ admits a complete hyperbolic metric of finite volume. Then the set of exceptional surgeries $\mathcal{E}_{K}=\{r \mid(K ; r)$ is not hyperbolic $\}$ is a finite set [50], [51], and $\mathcal{E}_{K}$ can be expressed as $\mathcal{R}_{K} \cup \mathcal{T}_{K} \cup \mathcal{S}_{K} \cup \mathcal{C}_{K}$, where

$\mathcal{R}_{K}=\{r \mid(K ; r)$ is reducible $\}$, $\mathcal{T}_{K}=\{r \mid(K ; r)$ is toroidal $\}$,

\footnotetext{
${ }^{1}$ Supported in part by Grant-in-Aid for Encouragement of Young Scientists (No. 11740051), The Ministry of Education, Science, Sports and Culture.

${ }^{2}$ Dedicated to Mitsuyoshi Kato on the occasion of his 60th birthday.
} 
$\mathcal{S}_{K}=\{r \mid(K ; r)$ is Seifert fibered $\}$ and

$\mathcal{C}_{K}=\{r \mid(K ; r)$ is a counter example to the geometrization conjecture $\}$.

By definition $\mathcal{S}_{K}$ contains a trivial surgery $\infty$. The geometrization conjecture [51] and the cabling conjecture [19] state that $\mathcal{C}_{K}=\emptyset$ and $\mathcal{R}_{K}=\emptyset$, respectively. Therefore it is expected that $\mathcal{E}_{K}=\mathcal{T}_{K} \cup \mathcal{S}_{K}$ for any hyperbolic knot $K$. The simplest knot satisfying $\mathcal{T}_{K} \neq \emptyset$ and $\mathcal{S}_{K} \neq\{\infty\}$ is the figureeight knot, for which we have $\mathcal{T}_{K}=\{0, \pm 4\}$ and $\mathcal{S}_{K}=\{\infty, \pm 1, \pm 2, \pm 3\}$ [50]. In this example, $\mathcal{T}_{K} \cap \mathcal{S}_{K}=\emptyset$. Recently Gordon and Luecke [25], Eudave-Muñoz [15, Proposition 4.5(1) and (3)] discovered an infinite family of hyperbolic knots $K$ with $\mathcal{T}_{K} \cap \mathcal{S}_{K} \neq \emptyset$.

For any hyperbolic knot $K$ it is known that $\left|\mathcal{T}_{K} \cap \mathcal{S}_{K}\right| \leq 7$, which is a consequence of the estimate of $\left|\mathcal{T}_{\mathcal{K}}\right|$ by Gordon [21], and that if $\mathcal{T}_{K} \cap \mathcal{S}_{K}$ is not empty, then it consists of only integers by Boyer and Zhang [6]. See also [22], [30] for surveys on Dehn surgery on knots.

Denote by $\operatorname{Diff}\left(S^{3}, K\right)$ the group of pairwise diffeomorphisms of $\left(S^{3}, K\right)$ (i.e., diffeomorphisms of $S^{3}$ which leave $K$ invariant). An element in $\operatorname{Diff}\left(S^{3}, K\right)$ having a finite order is called a symmetry of $K$. We regard two symmetries to be the same if they are conjugate by a diffeomorphism of $\left(S^{3}, K\right)$ isotopic to the identity map. We denote by $\operatorname{Diff}^{*}\left(S^{3}, K\right)$ the subgroup of $\operatorname{Diff}\left(S^{3}, K\right)$ consisting of elements which preserve an orientation of $S^{3}$. Then $\operatorname{Sym}^{*}(K)$ is defined as the group of pairwise isotopy classes of elements in $\operatorname{Diff}^{*}\left(S^{3}, K\right)$. For any hyperbolic knot $K$, it is known that $\operatorname{Sym}^{*}(K)$ is a finite group by Mostow-Prasad rigidity theorem [41], [43].

Each knot $K$ in [25], [15] is strongly invertible, hence $\operatorname{Sym}^{*}(K)$ contains a nontrivial element represented by the strong inversion. All the known examples suggest that if $\mathcal{S}_{K} \neq\{\infty\}$, then $\left|\operatorname{Sym}^{*}(K)\right| \geq 2$.

A relationship between symmetries of knots and Seifert fibered surgeries have been studied in [53], [35] and [36]. In the present paper we will consider these relations and put some further restriction on obtaining Seifert fiber spaces by surgeries on knots with symmetries.

Theorem 1.1 (Toroidal Seifert fibered surgeries). Let $K$ be a hyperbolic knot with $\left|\operatorname{Sym}^{*}(K)\right|>2$. Then $(K ; r)$ cannot be a toroidal Seifert fiber space for any $r$, and hence $\mathcal{T}_{K} \cap \mathcal{S}_{K}=\emptyset$.

Remark. (1) It should be emphasized that the condition $\left|\operatorname{Sym}^{*}(K)\right|>2$ does not imply either $\mathcal{T}_{K}=\emptyset$ or $\mathcal{S}_{K}=\{\infty\}$. In fact, the figure-eight knot has the property: $\left|\operatorname{Sym}^{*}(K)\right|>2, \mathcal{T}_{K} \neq \emptyset$ and $\mathcal{S}_{K} \neq\{\infty\}$.

(2) For satellite knots $K$, in general $\left|\operatorname{Sym}^{*}(K)\right|=\infty$ ([45]) and we will handle this case in Theorem 1.5. 
As a special case of Theorem 1.1 we have:

Corollary 1.2. Let $K$ be a hyperbolic knot with two strong inversions. Then $K$ has no toroidal Seifert fibered surgeries, hence $\mathcal{T}_{K} \cap \mathcal{S}_{K}=\emptyset$.

For homological reason, the base surface of a Seifert fiber surgery $(K ; r)$ (i.e., the quotient space of $(K ; r)$ by identifying each fiber to a point) is the 2 -sphere $S^{2}$ or the projective plane $\mathbb{R} P^{2}$. The following theorem deals with the latter case.

Theorem 1.3 (Projective Seifert fibered surgeries). Let $K$ be a hyperbolic knot with a symmetry which is not a strong inversion. Then $K$ has no surgery yielding a Seifert fiber space over $\mathbb{R} P^{2}$.

Remark. (1) There are many examples of such surgeries on hyperbolic knots with strong inversions. See [2], [6] for the case that the surgery manifold has a finite fundamental group, and [25], [15] for the case that the resulting manifold has an infinite fundamental group. Theorem 1.3 shows that these knots have no symmetries other than strong inversions.

(2) Dehn surgeries producing Seifert fiber spaces over $\mathbb{R} P^{2}$ are completely determined for torus knots ([40], [20]) and satellite knots [34].

Theorems 1.1, 1.3 can be combined with the work of Wang and Zhou [53] to deduce:

Corollary 1.4. Suppose that $(K ; r)(r \neq \infty)$ is a Seifert fiber space for a hyperbolic knot $K$ with $\left|\operatorname{Sym}^{*}(K)\right|>2$. Then the base surface must be $S^{2}$ and $(K ; r)$ has exactly three exceptional fibers.

Now let us turn to toroidal Seifert fibered surgeries on non-hyperbolic knots. As shown in [33], there are infinitely many satellite knots (with positive Gromov volume) with $\mathcal{T}_{K} \cap \mathcal{S}_{K} \neq \emptyset$. One can check that they are strongly invertible.

Theorem 1.5. Let $K$ be a non-hyperbolic knot with a symmetry which is not a strong inversion. If $(K ; r)$ is a toroidal Seifert fiber space, then $K$ is a trefoil knot $(r=0), T_{p, q} \sharp T_{p, q}(r=2 p q)$ or $T_{p, q} \sharp T_{-p, q}(r=0)$.

We then apply our results to surgeries on 2-bridge knots. In [8] Brittenham and $\mathrm{Wu}$ determined whether a given surgery on a 2-bridge knot is hyperbolic, reducible, toroidal, or atoroidal Seifert fibered. Note that 0surgery on a trefoil knot produces a toroidal Seifert fiber space. Since any 2-bridge knot is strongly invertible and admits a cyclic period 2 (see Section 
2 for definitions), $\operatorname{Sym}^{*}(K)$ contains two nontrivial elements represented by these symmetries. Thus Theorems 1.1 and 1.5 imply:

Corollary 1.6. Let $K$ be a 2-bridge knot which is not a trefoil knot. Then $\mathcal{T}_{K} \cap \mathcal{S}_{K}=\emptyset$.

Following [23] we shall say that a Seifert fiber space $M$ is of type $X\left(n_{1}, n_{2}, \ldots, n_{p}\right)$ if $M$ admits a Seifert fibration over $X$ with $p$ exceptional fibers of indices $n_{1}, n_{2}, \ldots, n_{p}\left(n_{i} \geq 2\right)$. Then $M$ is called small if $M$ is of type $S^{2}\left(n_{1}, n_{2}, \ldots, n_{p}\right)$ with $p \leq 3$. For convenience, we assume (without loss of generality) that $n_{1} \leq n_{2} \leq \cdots \leq n_{p}$ and if $M$ admits a Seifert fibration over $X$ without exceptional fiber, then we say that $M$ is of type $X(1)$ (by pretending that one regular fiber is an exceptional fiber of index 1).

Finally we apply the argument in the proof of Theorem 1.1 to surgeries yielding small Seifert fiber spaces.

Let $K$ be a knot with a cyclic period. If the period is greater than 2 , then it was previously known that $(K ; r)$ cannot be a Seifert fiber space except when $K$ is a torus knot or a cable of a torus knot ([35, Theorem 1.5, Proposition 5.6 and Added in proof]). If the period is 2 and $(K ; r)$ is a Seifert fiber space of type $X\left(n_{1}, n_{2}, \ldots, n_{p}\right)$, then $X=S^{2}$ ([36, Theorem 1.3]) and $p \geq 3$ ([53]); Furthermore if $p=3$, i.e., $(K ; r)$ is of type $S^{2}\left(n_{1}, n_{2}, n_{3}\right)$, then $\left(n_{i}, n_{j}\right)=1$ for some $i, j$ [36, Theorem 1.4]. By assuming further a strong invertibility, Corollary 1.4 implies that $p=3$ and we can put a stronger restriction on $n_{1}, n_{2}, n_{3}$.

Theorem 1.7 (Small Seifert fibered surgeries). Let $K$ be a strongly invertible knot with cyclic period 2.

(1) If $(K ; r)$ is a small Seifert fiber space of type $S^{2}\left(n_{1}, n_{2}, n_{3}\right)$ with $2<$ $n_{1}<n_{2}<n_{3}$, then $K$ is a torus knot or a cable of a torus knot.

(2) If $(K ; r)$ is a small Seifert fiber space of type $S^{2}\left(2, n_{2}, n_{3}\right)$ such that $\left(n_{2}, n_{3}\right) \neq 1$ and $\left(n_{2}, n_{3}\right) \neq(3,3)$, then $K$ is a torus knot or a cable of a torus knot.

In [37] we have shown that for a given triple $\left(n_{1}, n_{2}, n_{3}\right)$ such that $\left(n_{i}, n_{j}\right)=1$ for some $i, j$, there is a strongly invertible hyperbolic knot $K$ such that $(K ; r)$ is a Seifert fiber space of type $S^{2}\left(n_{1}, n_{2}, n_{3}\right)$. [35, Theorem 1.5, Proposition 5.6] and Theorem 1.7 show that most of them have no cyclic period. 
Acknowledgements - A part of this work is motivated by a discussion with Katura Miyazaki. I would like to thank him for useful discussions, especially for suggesting Lemma 5.2 and the use of covering space theory in the proof of Proposition 5.1. I would also like to thank the referee for careful reading and useful suggestions.

\section{Outline of the paper.}

Let $K$ be a nontrivial knot in $S^{3}$ and $\varphi: S^{3} \rightarrow S^{3}$ an orientation preserving periodic diffeomorphism of period $p \geq 2$ (i.e., $\varphi^{p}=$ id.) which satisfies $\varphi(K)=K$. We write $\operatorname{Fix}(\varphi)$ to denote the set of fixed points of $\varphi$. If $\operatorname{Fix}(\varphi) \neq \emptyset$, by the positive answer to the Smith conjecture [39], $\varphi$ is a rotation of $S^{3}$ about the unknotted circle $\operatorname{Fix}(\varphi)$.

$\emptyset$.

If $\operatorname{Fix}(\varphi) \neq \emptyset$, there are two possibilities: $\operatorname{Fix}(\varphi) \cap K \neq \emptyset$ or $\operatorname{Fix}(\varphi) \cap K=$

Definition (strong inversion). If $\operatorname{Fix}(\varphi) \neq \emptyset$ and $\operatorname{Fix}(\varphi) \cap K \neq \emptyset$, then $K$ is said to be strongly invertible. In this case $\operatorname{Fix}(\varphi)$ intersects $K$ in two points and $\varphi$ reverses an orientation of $K$, and hence the period $p=2$. Such an involution is called a strong inversion of $K$.

Definition (cyclic period). If $\operatorname{Fix}(\varphi) \neq \emptyset$ and $\operatorname{Fix}(\varphi) \cap K=\emptyset$, then we say that $K$ has a cyclic period $p$.

Next let us assume that $\operatorname{Fix}(\varphi)=\emptyset$. We have two possibilities: $\operatorname{Fix}\left(\varphi^{i}\right)=$ $\emptyset$ for $i=1, \cdots, p-1$, or $\operatorname{Fix}\left(\varphi^{i}\right) \neq \emptyset$ for some $i(1<i<p)$.

Definition (free period). If $\operatorname{Fix}\left(\varphi^{i}\right)=\emptyset$ for $i=1, \cdots, p-1$, then we say that $K$ has a free period $p$.

Definition (semi-free period). If $\operatorname{Fix}(\varphi)=\emptyset$ but $\operatorname{Fix}\left(\varphi^{i}\right) \neq \emptyset$ for some $i$ such that $1<i<p$, then we say that $K$ has a semi-free period $p$. Since $\varphi$ preserves an orientation of $K$, so does $\varphi^{i}$ and $\varphi^{i}$ gives a cyclic period of $K$.

Suppose that $K$ is a hyperbolic knot with $\left|\operatorname{Sym}^{*}(K)\right|>2$. Then from [5, Theorem 2.1(b)] or [45, Theorem 3.1] we have a finite group $\Gamma \subset \operatorname{Diff} *\left(S^{3}, K\right)$ which realizes $\operatorname{Sym}^{*}(K)$. If $\Gamma$ contains a diffeomorphism $\varphi$ of period $p>2$, then $\varphi$ gives a cyclic, free or semi-free period $p>2$. If $\Gamma$ contains no such a diffeomorphism, then each nontrivial element of $\Gamma$ has order 2 (i.e., each element is an involution). Since $|\Gamma|>2, \Gamma$ contains involutions $\varphi$ and $\psi$; neither $\varphi$ nor $\psi$ gives a semi-free period.

Lemma 2.1. At least one of $\varphi$ and $\psi$ is a strong inversion. 
Proof. To the contrary suppose that neither $\varphi$ nor $\psi$ is a strong inversion. Then both involutions $\varphi$ and $\psi$ preserve an orientation of $K$. This then implies that the finite group $\left\langle\left.\varphi\right|_{K},\left.\psi\right|_{K}\right\rangle$ acts freely on $K$. Thus $\left\langle\left.\varphi\right|_{K},\left.\psi\right|_{K}\right\rangle$ is a finite cyclic group. The Smith conjecture [39] implies that $\langle\varphi, \psi\rangle$ is isomorphic to $\left\langle\left.\varphi\right|_{K},\left.\psi\right|_{K}\right\rangle$. Thus $\langle\varphi, \psi\rangle$ is also cyclic, which implies that $\varphi=\psi$, because $\varphi$ and $\psi$ are involutions. This is a contradiction. $\square$ (Lemma 2.1)

Following the above observation, we shall say that a knot $K$ has Type $C, F$, or $S F$ symmetry if $K$ has a cyclic, free, or semi-free period $p>2$, and that $K$ has Type $I-I, I-C$, or $I-F$ symmetry if $K$ has two (distinct) involutions $\varphi, \psi$ generating a finite subgroup of $\operatorname{Diff}^{*}\left(S^{3}, K\right)$ such that both $\varphi$ and $\psi$ are strong inversions, $\varphi$ is a strong inversion and $\psi$ gives a cyclic period 2, or $\varphi$ is a strong inversion and $\psi$ gives a free period 2, respectively.

Lemma 2.2. Suppose that $K$ has Type I-I symmetry. Then $K$ admits also Type C, F, I-C or I-F symmetry.

Proof. By definition $K$ has two distinct strong inversions $\varphi$ and $\psi$, which generate a finite group $\Gamma$. Put $\tau=\varphi \circ \psi$, which is also periodic, because $\Gamma$ is finite. Note that $\tau$ is not the identity map, because $\varphi \neq \psi$, and that it preserves an orientation of $K$. If $\tau^{i}$ is fixed point free for all $i$ less than the period of $\tau$, then $\tau$ gives a free period of $K$. Thus $K$ has Type $\mathrm{F}$ or Type I-F symmetry depending on whether the period of $\tau$ is greater than 2 or exactly 2. If $\tau^{i}$ has a fixed point for some $i$ less than the period of $\tau$, then $\tau^{i}$ gives a cyclic period of $K$. Thus $K$ has Type C or Type I-C symmetry depending on whether the period of $\tau^{i}$ is greater than 2 or exactly $2 . \quad \square$ (Lemma 2.2)

We summarize the above observation in the following lemma.

Lemma 2.3. Let $K$ be a hyperbolic knot with $\left|\operatorname{Sym}^{*}(K)\right|>2$. Then $K$ has Type $C, F, S F, I-C$ or I-F symmetry.

For knots with Type C symmetry, we recall:

Theorem 2.4 ([35]). Let $K$ be a knot with a cyclic period $p>2$. If $(K ; r)$ is a Seifert fiber space, then $K$ is a torus knot or a cable of a torus knot. In particular, a hyperbolic knot $K$ with Type $C$ symmetry has no Seifert fibered surgery.

In Section 4, we will study Dehn surgeries on hyperbolic, freely periodic knots and prove: 
Theorem 4.5. Let $K$ be a hyperbolic, freely periodic knot. Then $(K ; r)$ cannot be a Seifert fiber space over $\mathbb{R} P^{2}$.

Theorem 4.10. Let $K$ be a hyperbolic knot with Type F symmetry, i.e., a free period $p>2$. Then $(K ; r)$ cannot be a toroidal Seifert fiber space.

From Theorems 2.4 and 4.10, we can deduce:

Theorem 4.15. Let $K$ be a hyperbolic knot with Type SF symmetry, i.e., a semi-free period $p>2$. Then $(K ; r)$ cannot be a toroidal Seifert fiber space.

Section 5 is devoted to a study of Seifert fibered surgeries on strongly invertible knots. We will study surgeries on hyperbolic knots with Type I-C or I-F in Sections 6-9. In Section 9, we will prove:

Theorem 9.1. Let $K$ be a hyperbolic knot with Type I- $C$ symmetry. Then $(K ; r)$ cannot be a toroidal Seifert fiber space.

Theorem 9.2. Let $K$ be a hyperbolic knot with Type I-F symmetry. Then $(K ; r)$ cannot be a toroidal Seifert fiber space.

In Section 10 we will prove Theorem 1.5. A proof of Theorem 1.3 will be completed in Section 11. In the final section Section 12, we will study Dehn surgeries yielding small Seifert fiber spaces and prove Theorem 1.7 by applying some results in Sections 5-8.

Proof of Theorem 1.1. Let $K$ be a hyperbolic knot with $\left|\operatorname{Sym}^{*}(K)\right|>$ 2 . Then by Lemma 2.3 , the theorem can be obtained by putting together Theorems 2.4, 4.10, 4.15, 9.1 and 9.2.

$\square$ (Theorem 1.1)

Proof of Corollary 1.2. Let $K$ be a hyperbolic knot with two strong inversions $\varphi$ and $\psi$. Denote by $[\varphi],[\psi] \in \operatorname{Sym}^{*}(K)$ the pairwise isotopy classes of $\varphi, \psi$, respectively. Both $[\varphi]$ and $[\psi]$ have order 2 , in particular these are nontrivial in $\operatorname{Sym}^{*}(K)$. Assume for a contradiction that $[\varphi]=[\psi] \in \operatorname{Sym}^{*}(K)$. Then by $[5$, Theorem 2.1 (c)] ([52, Corollary 7.2]) $\varphi$ and $\psi$ are conjugate by a diffeomorphism of $\left(S^{3}, K\right)$ isotopic to the identity map (see also [4], [10]). This contradicts the assumption. It follows that $\operatorname{Sym}^{*}(K)$ contains more than two elements and the result follows from Theorem 1.1. $\square$ (Corollary $1.2)$

Proof of Corollary 1.4. Assume that $K$ is a hyperbolic knot with $\left|\operatorname{Sym}^{*}(K)\right|>2$. Then Lemma 2.3 implies that $K$ admits a symmetry which is not a strong inversion. By Theorem 1.3 the base surface of $(K ; r)$ must be the 2-sphere, and by Theorem 1.1 the number of exceptional fibers is less than four, for otherwise $(K ; r)$ contains a vertical incompressible torus. On the other hand, the number of exceptional fibers is greater than two by [53], because $K$ has a cyclic or free period by Lemma $2.3 . \quad \square$ (Corollary 1.4 ) 


\section{Isomorphisms of base orbifolds and detecting fibers.}

Let $(K ; r)$ be a Seifert fiber space and $\bar{\varphi}:(K ; r) \rightarrow(K ; r)$ an orientation preserving periodic diffeomorphism with period $p \geq 2$, which has a (non-empty, possibly disconnected) 1-dimensional fixed point set $\operatorname{Fix}(\bar{\varphi})$. (In practice, we shall consider a situation where $\bar{\varphi}$ is induced from an orientation preserving periodic diffeomorphism $\varphi$ of $\left(S^{3}, K\right)$.)

Assume here that $(K ; r)$ has a Seifert fibration preserved by $\bar{\varphi}$. In most cases, such an invariant Seifert fibration exists. In fact, from [31] and [35, Proposition 5.7] we have the following.

Lemma 3.1. Suppose that $K$ is a nontrivial knot which is not a trefoil knot and $(K ; r)$ is a Seifert fiber space.

(1) If $\pi_{1}((K ; r))$ is infinite, then for any finite group $G$ acting on $(K ; r)$, there exists a $G$-invariant Seifert fibration of $(K ; r)$.

(2) If $\pi_{1}((K ; r))$ is finite and $g$ is a periodic diffeomorphism with a 1dimensional fixed point set, then $(K ; r)$ admits a $\langle g\rangle$-invariant Seifert fibration.

Proof. If $(K ; r)$ is reducible, then since $(K ; r)$ is Seifert fibered and $H_{1}((K ; r))$ is cyclic, $(K ; r) \cong S^{2} \times S^{1}$ [27, VI.7.Lemma]. Then $K$ is a trivial knot and $r=0$ [17, Corollary 8.3]. So we may assume $(K ; r)$ is irreducible.

(1) Since $(K ; r)$ is irreducible and $\pi_{1}((K ; r))$ is infinite, $(K ; r)$ possesses a geometric structure modelled on $\mathbb{E}^{3}, \mathrm{Nil}, H^{2} \times \mathbb{R}$ or $\widetilde{S L_{2} \mathbb{R}}$ (see [47]). If $(K ; r)$ possesses the $\mathbb{E}^{3}$-geometry, then $K$ is a trefoil knot [35, Lemma 5.4], contradicting the assumption on $K$.

Now we assume that $(K ; r)$ possesses a geometric structure modelled on Nil, $H^{2} \times \mathbb{R}$ or $\widetilde{S L_{2} \mathbb{R}}$. Under these geometries it follows from a result of Meeks-Scott [31] we can choose a $G$-invariant Seifert fibration of $(K ; r)$ (see also [31, p.289]).

(2) Since $g$ has a 1-dimensional fixed point set and $(K ; r)$ has a finite fundamental group, the Orbifold Theorem ([4], [10]) implies that $(K ; r)$ possesses an $S^{3}$-geometry and $g$ is an isometry. Applying [35, Lemma 5.7], we have a $\langle g\rangle$-invariant Seifert fibration of $(K ; r)$.

$\square($ Lemma 3.1)

The base surface of $(K ; r)$ has naturally an orbifold structure so that a cone point of index $n$ is the image of an exceptional fiber of index $n$. Such a 2-orbifold $B$ is referred to as the base orbifold of $(K ; r)$ and its underlying space is denoted by $|B|$. 
Since $\bar{\varphi}$ preserves the Seifert fibration, it induces an orbifold isomorphism $\hat{\varphi}$ on the base orbifold $B$ so that the following diagram commutes. Here $\pi:(K ; r) \rightarrow B$ denotes the natural projection with respect to the Seifert fibration of $(K ; r)$.

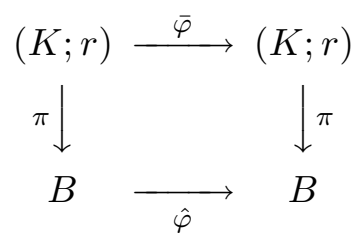

Note that $\hat{\varphi}$ has also period at most $p$ and it is conjugate to an orthogonal transformation on $S^{2}$ ([13], [9]), i.e., the identity map, a rotation around the North-South axis, a reflection along the equator, or the composition of a rotation around the North-South axis with a reflection along the equator. For simplicity, we say that $\hat{\varphi}$ is a rotation (resp. a reflection) if it is conjugate to a rotation around the North-South axis (resp. a reflection along the equator).

Lemma 3.2. Suppose that $\operatorname{Fix}(\bar{\varphi}) \neq \emptyset$.

(1) If $\hat{\varphi}$ preserves an orientation of the base surface $|B|=S^{2}$, then each component of $\operatorname{Fix}(\bar{\varphi})$ is a Seifert fiber in $(K ; r)$.

(2) If $\hat{\varphi}$ is the identity map on the base surface $|B|=\mathbb{R} P^{2}$, then each component of $\operatorname{Fix}(\bar{\varphi})$ is a Seifert fiber in $(K ; r)$.

Proof. Let $L$ be a component of $\operatorname{Fix}(\bar{\varphi})$ and $t$ a fiber intersecting $L$. Since $\bar{\varphi}$ preserves the Seifert fibration, we obtain a periodic map $\left.\bar{\varphi}\right|_{t}: t \rightarrow t$, which has a nonempty fixed point set $t \cap L$. Hence $\left.\bar{\varphi}\right|_{t}$ is the identity map or a reflection. If $\left.\bar{\varphi}\right|_{t}=\mathrm{id}$, , then $t=L$ as desired.

Suppose for a contradiction that $\left.\bar{\varphi}\right|_{t}$ is a reflection. Let $N(t)$ be a $\langle\bar{\varphi}\rangle$ invariant fibered tubular neighborhood of $t$. Since $\bar{\varphi}$ is orientation preserving, but reverses an orientation of $t, \bar{\varphi}$ reverses an orientation of a meridian of $N(t)$. Hence $\left.\hat{\varphi}\right|_{\partial \pi(N(t))}$ reverses an orientation of $\partial \pi(N(t))$. This contradicts the assumptions in (1), (2).

$\square$ (Lemma 3.2)

Furthermore, we have:

Lemma 3.3. If a component of $\operatorname{Fix}(\bar{\varphi})$ is a regular fiber, then the induced isomorphism $\hat{\varphi}: B \rightarrow B$ is not the identity map. 
Proof. Let us assume that a component $L_{\varphi}^{\prime}$ of $\operatorname{Fix}(\bar{\varphi})$ is a regular fiber. Let $N\left(L_{\varphi}^{\prime}\right)$ be a fibered neighborhood of $L_{\varphi}^{\prime}$ which is invariant under $\langle\bar{\varphi}\rangle$, and let $D$ be a $\langle\bar{\varphi}\rangle$-equivariant meridian disk of $N\left(L_{\varphi}^{\prime}\right)([32])$. Since $L_{\varphi}^{\prime} \cap D \neq \emptyset$, $\bar{\varphi}(D)=D$. Let us choose a regular fiber $t$ intersecting $\partial D$ transversely. Since $L_{\varphi}^{\prime}$ is a regular fiber, the algebraic intersection number of $t$ and $\partial D$ is \pm 1 .

To the contrary suppose that $\hat{\varphi}$ is the identity map on $B$. Then $\bar{\varphi}(t)=t$ and $\bar{\varphi}$ induces a periodic diffeomorphism $\left.\bar{\varphi}\right|_{t}: t \rightarrow t$ of period $p \geq 2$. Since $\bar{\varphi}(\partial D)=\partial D$, we have $\bar{\varphi}(t \cap \partial D)=t \cap \partial D$. Thus $\bar{\varphi}$ acts on $t \cap \partial D$, and since $\bar{\varphi}$ preserves an orientation of $\partial N\left(L_{\varphi}^{\prime}\right)$, each $\langle\bar{\varphi}\rangle$-orbit in $t \cap \partial D$ consists of $p$ points of the same sign. This implies the algebraic intersection number of $t$ and $\partial D$ is a multiple of $p \geq 2$, a contradiction. It follows that $\hat{\varphi}$ is not the identity map.

$\square$ (Lemma 3.3)

\section{Seifert fibered surgeries on freely periodic knots.}

Assume that $K$ is a freely periodic knot with period $p \geq 2$ and $f: S^{3} \rightarrow S^{3}$ a periodic diffeomorphism giving the free period $p$ of $K$. Let us choose an $\langle f\rangle$ invariant tubular neighborhood $N(K)$ and an $\langle f\rangle$-equivariant simple loop $\alpha$ on $\partial N(K)$ representing $r$. Then we can extend $\left.f\right|_{S^{3}-\operatorname{int} N(K)}$ over $(K ; r)$ periodically to obtain an orientation preserving diffeomorphism $\bar{f}:(K ; r) \rightarrow$ $(K ; r)$ of period $p$. If $f^{i}(\alpha)=\alpha\left(\operatorname{resp} . f^{i}(\alpha) \cap \alpha=\emptyset\right)$, then $\operatorname{Fix}\left((\bar{f})^{i}\right)=K^{*}$, where $K^{*}$ denotes the dual knot of $K$, i.e., the core of the filling solid torus $\left(\right.$ resp. $\left.\operatorname{Fix}\left((\bar{f})^{i}\right)=\emptyset\right)$. Thus for each $i(1 \leq i \leq p-1)$ we have $\operatorname{Fix}\left((\bar{f})^{i}\right)=\emptyset$ or $K^{*}$.

Assume further that $(K ; r)$ is a Seifert fiber space whose Seifert fibration is preserved by $\bar{f}$. Then we obtain a periodic isomorphism $\hat{f}$ of the base orbifold $B$. Recall that $|B|=S^{2}$ or $\mathbb{R} P^{2}$.

\subsection{Preliminary lemmas.}

Lemma 4.1. Suppose that $\operatorname{Fix}(\bar{f})=K^{*}$. If $\hat{f}$ preserves an orientation of the base surface $|B|=S^{2}$ or $\hat{f}$ is the identity map on $|B|=\mathbb{R} P^{2}$, then $K$ is a torus knot.

Proof. Under the assumption in Lemma 4.1, Lemma 3.2 asserts that $K^{*}$ is a fiber in $(K ; r)$. Thus $S^{3}-K \cong(K ; r)-K^{*}$ is Seifert fibered, and hence $K$ is a torus knot in $S^{3}$.

$\square$ (Lemma 4.1) 
Lemma 4.2. If $K$ is not a torus knot and $\operatorname{Fix}(\bar{f})=K^{*}$, then the period $p$ is 2 .

Proof. If $K^{*}$ is a fiber in $(K ; r)$, then as in the proof of Lemma 4.1 we see that $K$ is a torus knot, contradicting the assumption. Therefore $K^{*}$ is not a fiber in $(K ; r)$. Let $t$ be a fiber intersecting $K^{*}$. Then $\bar{f}(t)=t$ and we have a periodic diffeomorphism $\left.\bar{f}\right|_{t}: t \rightarrow t$. Since $\left.\bar{f}\right|_{t}$ fixes a point in $t \cap K^{*}$, it is a reflection and $\operatorname{Fix}\left((\bar{f})^{2}\right) \supset t\left(\neq K^{*}\right)$. This implies that the period $p$ is 2 .

Let us suppose that the action of $\langle\bar{f}\rangle$ on $(K ; r)$ is free, i.e., $\operatorname{Fix}\left(\bar{f}^{i}\right)=\emptyset$ for $i=1, \cdots, p-1$.

Definition ( $\hat{f}$-essential loop). A simple loop $c$ on the base orbifold $B$ $\left(|B|=S^{2}\right.$ or $\left.\mathbb{R} P^{2}\right)$ is called $\hat{f}$-essential if

(1) $c$ is two-sided, and hence it separates $B$;

(2) $c$ does not meet any cone point and does not bound a diskal orbifold (i.e., a disk with at most one cone point); and

(3) For each $i, \hat{f}^{i}(c)=c$ or $\hat{f}^{i}(c) \cap c=\emptyset$; if $\hat{f}^{i}(c)=c$, then $\hat{f}^{i}$ does not exchange the sides of $c$.

Lemma 4.3. Suppose that $K$ is a hyperbolic knot and $\operatorname{Fix}\left(\bar{f}^{i}\right)=\emptyset$ for $i=1, \ldots, p-1$. Then there is no $\hat{f}$-essential loop on the base orbifold $B$ of $(K ; r)$.

Proof. To the contrary suppose that there exists an $\hat{f}$-essential loop $c$ on $B$. Then we have an $\langle\bar{f}\rangle$-equivariant vertical incompressible torus $T=\pi^{-1}(c) \subset$ $(K ; \gamma)$, and by [7, Corollary 1.7] $\gamma$ is an integral slope.

The free action of $\langle f\rangle$ on $S^{3}$ induces a $p$-fold (unbranched) cyclic covering $\rho: S^{3} \rightarrow S^{3} /\langle f\rangle ; \pi_{1}\left(S^{3} /\langle f\rangle\right) \cong \mathbb{Z}_{p}$. Then we have a new knot $K_{f}=\rho(K)$ in $S^{3} /\langle f\rangle$. Similarly, since the action of $\langle\bar{f}\rangle$ on $(K ; \gamma)$ is also free, we have a $p$ fold (unbranched) cyclic covering $\bar{\rho}:(K ; \gamma) \rightarrow(K ; \gamma) /\langle\bar{f}\rangle$. Since the Seifert fibration is invariant under the action of $\langle\bar{f}\rangle,(K ; r) /\langle\bar{f}\rangle$ admits an induced Seifert fibration so that the covering map $\bar{\rho}$ preserves their Seifert fibrations. Furthermore, $(K ; r) /\langle\bar{f}\rangle$ contains a vertical separating incompressible torus $\bar{\rho}(T)$. (Note that by the choice of $T, \bar{\rho}(T)$ is a torus, not a Klein bottle.) In particular, since any small Seifert fiber space cannot contain a separating incompressible torus ([27, VI.13. Example]), $(K ; r) /\langle\bar{f}\rangle$ is not a small Seifert fiber space.

We remark here that the quotient manifold $(K ; \gamma) /\langle\bar{f}\rangle$ can be regarded also as the manifold obtained from $S^{3} /\langle f\rangle$ by $\gamma_{f}$-surgery on $K_{f}\left(\subset S^{3} /\langle f\rangle\right)$ for some slope $\gamma_{f}$, which we denote by $S^{3} /\langle f\rangle\left(K_{f} ; \gamma_{f}\right)$. 
Thus we have the diagram:

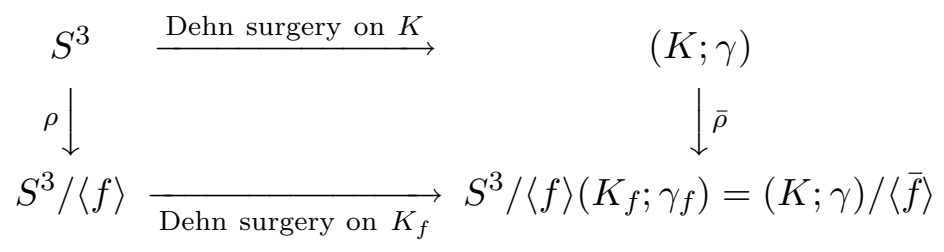

Claim 4.4. The distance, i.e., the minimal geometric intersection number, between the surgery slope $\gamma_{f}$ and the meridional slope $\mu_{f}$ is $p$.

Proof of Claim 4.4. Since $\operatorname{Fix}\left(\bar{f}^{i}\right)=\emptyset$ for $1 \leq i \leq p-1$, the $\langle f\rangle$-equivariant loop $\alpha$ representing the surgery slope $\gamma$ satisfies that $\alpha, \bar{f}(\alpha), \cdots, \bar{f}^{p-1}(\alpha)$ are mutually disjoint loops. Thus $\left.\rho\right|_{\alpha}: \alpha \rightarrow \rho(\alpha)$ is a diffeomorphism, and $\left.\rho\right|_{K}: K \rightarrow K_{f}$ is a $p$-fold covering. Since $\gamma$ is an integral slope, $\alpha$ is homologous to $K$. Hence $\rho(\alpha)$, which is a simple loop representing $\gamma_{f}$, is homologous to $p K_{f}$. It follows that the distance between $\gamma_{f}$ and $\mu_{f}$ is $p$ as desired.

$\square($ Claim 4.4)

Since $K$ is a hyperbolic knot in $S^{3}$ and $S^{3}-K \rightarrow S^{3} /\langle f\rangle-K_{f}$ is unbranched finite covering, $S^{3} /\langle f\rangle-K_{f}$ is irreducible, neither toroidal nor Seifert fibered. Thus $K_{f}$ is also a hyperbolic knot in $S^{3} /\langle f\rangle([39])$. Applying [7, Theorem 1.5] to the cyclic surgery slope $\mu_{f}$ and the toroidal Seifert fibered surgery slope $\gamma_{f}$, we see that their distance is 1 . Hence by Claim 4.4 the period $p=1$, a contradiction.

$\square($ Lemma 4.3)

\subsection{Seifert fibered surgeries over $\mathbb{R} P^{2}$.}

The goal in this subsection is to prove the following result, in which we allow the case where $(K ; r)$ has a finite fundamental group.

Theorem 4.5. Let $K$ be a hyperbolic, freely periodic knot. Then $(K ; r)$ cannot be a Seifert fiber space over $\mathbb{R} P^{2}$.

Proof. Assume for a contradiction that $(K ; r)$ is a Seifert fiber space over $\mathbb{R} P^{2}$. Then it contains a Klein bottle, and [24] shows that $r$ is an integer $m$. (This fact can also be deduced from [6] if $\left|\pi_{1}\right|<\infty$ and [7] if $\left|\pi_{1}\right|=\infty$.) Recall from [36] that: 
Lemma $4.6([36])$. Suppose that $(K ; m)$ is a Seifert fiber space of type $\mathbb{R} P^{2}\left(\alpha_{1}, \ldots, \alpha_{k}\right)$. Then $m=4 x$ for some nonzero integer $x$ and $\alpha_{i} \neq \alpha_{j}$ for $i \neq j$.

Proof. The fundamental group of $(K ; m)$ has a presentation $([27])$

$$
\left\langle a, c_{1}, \ldots, c_{k}, h \mid a h a^{-1}=h^{-1}, c_{i} h c_{i}^{-1}=h, c_{i}^{\alpha_{i}}=h^{\beta_{i}}, h^{b}=a^{2} c_{1} \cdots c_{k}\right\rangle
$$

for some integers $\beta_{i}$ and $b$; possibly $\alpha_{1}=1$ but $\alpha_{i} \geq 2$ for $i \geq 2$.

A computation of the determinant of the presentation matrix of the abelianization shows that $\left|H_{1}((K ; m))\right|=4 \alpha_{1} \ldots \alpha_{k}$, i.e., $|m|=4 \alpha_{1} \ldots \alpha_{k}$. Putting $x=\alpha_{1} \cdots \alpha_{k}$, which is a nonzero integer, the first assertion is proved.

To prove the second assertion, assume for a contradiction that there is a pair of cone points of the same index; without loss of generality we may assume that $\alpha_{1}=\alpha_{2}=\alpha \geq 2$. Putting $h=1, c_{3}=\cdots=c_{k}=1$ and then abelianizing the group, we obtain a non-cyclic group $\mathbb{Z}_{\alpha} \oplus \mathbb{Z}_{2 \alpha}$. This contradicts the fact that $H_{1}((K ; m))$ is cyclic.

$\square$ (Lemma 4.6)

Let $f$ be a periodic diffeomorphism of $\left(S^{3}, K\right)$ giving the free period of $K$. By taking some power $f^{x}$ of $f$, if necessary, we may assume that $f^{x}$ has prime period $p^{\prime}$ and $\operatorname{Fix}\left(\overline{f^{x}}\right)=K^{*}$ or $\operatorname{Fix}\left(\left(\overline{f^{x}}\right)^{i}\right)=\emptyset$ for $i=1, \cdots, p^{\prime}-1$. For notational simplicity, we still use $f$ and $p$ to denote $f^{x}$ and $p^{\prime}$ respectively so that we suppose that $f$ gives a free period of $K$ of prime period $p$.

We distinguish two cases depending on whether $(K ; m)$ contains at least two exceptional fibers or not.

Case $1:(K ; m)$ contains at least two exceptional fibers.

Note that $(K ; m)$ contains a vertical incompressible torus. In this case from Lemma $3.1(1)$, we have an $\langle\bar{f}\rangle$-invariant Seifert fibration of $(K ; m)$ with the natural projection $\pi:(K ; m) \rightarrow \mathbb{R} P^{2}$.

Let us divide into two cases: $\operatorname{Fix}(\bar{f})=K^{*}$ or $\operatorname{Fix}\left(\bar{f}^{i}\right)=\emptyset$ for $i=$ $1, \cdots, p-1$.

First assume that $\operatorname{Fix}(\bar{f})=K^{*}$. Since $K$ is hyperbolic, by Lemma 4.2 the period $p$ is 2 . Let $\alpha \subset \partial E(K)$ be an $\langle f\rangle$-equivariant simple loop representing the surgery slope $m$. Recall from Lemma 4.6 that $m$ is an even integer.

Claim 4.7. $f(\alpha) \cap \alpha=\emptyset$.

Proof. Since $f$ is a free involution, from the argument in [26, p.180] we see that there is no $\langle f\rangle$-invariant preferred longitude, and hence that $\left.f\right|_{N(K)}$ is an involution of Type $P_{4}$ described in [26]. Therefore we can choose a diagonal loop $\delta$ representing [meridian $]+[$ longitude $]$ so that $\delta$ is invariant 
under $\langle f\rangle$. This implies that if $m$ is even (resp. odd), then $f(\alpha) \cap \alpha=\emptyset$ (resp. $f(\alpha)=\alpha$ ).

$\square$ (Claim 4.7)

Claim 4.7 would imply that $\operatorname{Fix}(\bar{f})=\emptyset$. This contradicts the assumption.

Next suppose that $\operatorname{Fix}\left(\bar{f}^{i}\right)=\emptyset$ for $i=1, \cdots, p-1$. Assume first that $\hat{f}: \mathbb{R} P^{2} \rightarrow \mathbb{R} P^{2}$ is the identity map. Then since $B$ contains at least two exceptional fibers, we have an $\hat{f}$-essential loop on $B$, contradicting Lemma 4.3. Thus $\hat{f}$ is not the identity map. We distinguish two cases: the period of $\hat{f}$ is 2 or greater than 2 .

First suppose that $\hat{f}$ has period 2 . Then $\operatorname{Fix}(\hat{f})$ is empty or else consists of a point $P_{f}$ and a 1-sided simple loop $C_{f}([48, \mathrm{p} .414])$. Let us suppose that $\operatorname{Fix}(\hat{f})=\emptyset$. Since $B$ contains at least two cone points, we can choose one of them, say $x \in B$. Then there are two cone points $x$ and $\hat{f}(x)$ of the same index, which would be impossible by Lemma 4.6.

Next we consider the case where $\operatorname{Fix}(\hat{f})$ consists of a point $P_{f}$ and a 1-sided simple loop $C_{f}$. Let $x$ be a (regular) point in $C_{f}$, and let $D$ be an $\langle\hat{f}\rangle$-invariant disk neighborhood of $x$. Then $\hat{f}$ is a reflection on $D$. Therefore $\bar{f}$ reverses an orientation of the fiber $t=\pi^{-1}(x)$. Thus $\left.\bar{f}\right|_{t}$ fixes two points in $t$, and hence $\operatorname{Fix}(\bar{f}) \neq \emptyset$, contradicting the assumption.

Finally we consider the case where the period of $\hat{f}$ is greater than 2 .

Claim 4.8. If the period of $\hat{f}$ is greater than 2 , then $\operatorname{Fix}(\hat{f})$ consists of at most one point $P_{f}$.

Proof. To prove the claim, we suppose for a contradiction that $\operatorname{Fix}(\hat{f})$ contains at least two points $P_{f}$ and $Q_{f}$.

Consider the universal cover $q: S^{2} \rightarrow \mathbb{R} P^{2}$, and put $q^{-1}\left(P_{f}\right)=\{\tilde{x}, \tilde{y}\}$. Let $\tilde{f}: S^{2} \rightarrow S^{2}$ be a lift of $\hat{f}: \mathbb{R} P^{2} \rightarrow \mathbb{R} P^{2}$ such that $\tilde{f}(\tilde{x})=\tilde{x}$. Since the period of $\tilde{f}$, which coincides with that of $\hat{f}$, is greater than 2 and $\tilde{f}$ has a fixed point, it is a rotation of $S^{2}$. Since $\hat{f}\left(P_{f}\right)=P_{f}$ and $q(\tilde{y})=P_{f}, \tilde{f}(\tilde{y})$ also covers $P_{f}$, hence $\tilde{f}(\tilde{y})=\tilde{x}$ or $\tilde{y}$. Since $\tilde{f}(\tilde{x})=\tilde{x}$, we have $\tilde{f}(\tilde{y})=\tilde{y}$; thus $\operatorname{Fix}(\tilde{f})=\{\tilde{x}, \tilde{y}\}$. Let $\tilde{z}$ be a point in $S^{2}$ such that $q(\tilde{z})=Q_{f}$. Since $Q_{f} \neq P_{f}$, $\tilde{z} \notin \operatorname{Fix}(\tilde{f})$ and $\langle\tilde{f}\rangle$-orbit of $\tilde{z}$ consists of more than two points. On the other hand, since $\hat{f}\left(Q_{f}\right)=Q_{f}$, any point in $\langle\tilde{f}\rangle$-orbit of $\tilde{z}$ covers the point $Q_{f}$, hence $\langle\tilde{f}\rangle$-orbit of $\tilde{z}$ consists of two points, a contradiction. $\square$ (Claim 4.8)

By Claim $4.8, \operatorname{Fix}(\hat{f})$ consists of at most one point, while $B$ contains at least two cone points, because that $(K ; m)$ contains at least two exceptional fibers. It follows that there is a cone point $x$ which is not in $\operatorname{Fix}(\hat{f})$. Then there are two cone points having the same index. This contradicts Lemma 4.6. It follows that $(K ; m)$ cannot be a Seifert fiber space over $\mathbb{R} P^{2}$ with at least two exceptional fibers. 
Case $2:(K ; m)$ contains at most one exceptional fiber.

Since $(K ; m)$ is a Seifert fiber space over $\mathbb{R} P^{2}$ with at most one exceptional fiber, which is not $\mathbb{R} P^{3} \sharp \mathbb{R} P^{3},(K ; m)$ admits also a Seifert fibration over $S^{2}$ with at most three exceptional fibers of indices $2,2, x(\geq 1)$. Since $(K ; m) \nRightarrow S^{2} \times S^{1}$, it has a finite fundamental group.

We divide into two cases: $\operatorname{Fix}(\bar{f})=K^{*}$ or $\operatorname{Fix}\left(\bar{f}^{i}\right)=\emptyset$ for $i=1, \cdots, p-1$.

Let us assume that $\operatorname{Fix}(\bar{f})=K^{*}$. Since $\operatorname{dimFix}(\bar{f})=1$, by Lemma 3.1 (2) an $\langle\bar{f}\rangle$-invariant Seifert fibration of $(K ; m)$ exists. Then the result follows by applying the same argument in Case 1 .

Now suppose that $\operatorname{Fix}\left(\bar{f}^{i}\right)=\emptyset$ for $i=1, \cdots, p-1$. As in the proof of Lemma $4.3, K_{f}$ is a hyperbolic knot in the quotient manifold $S^{3} /\langle f\rangle$ whose fundamental group is a cyclic group $\mathbb{Z}_{p}$. Furthermore, since $(K ; m)$ has a finite fundamental group and $\bar{\rho}:(K ; m) \rightarrow(K ; m) /\langle\bar{f}\rangle$ is (unbranched) $p$-fold covering, $(K ; m) /\langle\bar{f}\rangle$ has also a finite fundamental group.

It should be noted here that since $\pi_{1}((K ; m))$ is finite and the action of $\langle\bar{f}\rangle$ on $(K ; m)$ is free, we cannot apply either Meeks-Scott's result [31, Theorem 2.2] or [35, Lemma 5.7].

Claim 4.9. $(K ; m) /\langle\bar{f}\rangle$ is a lens space $\left(\not S^{2} \times S^{1}\right)$ or a prism manifold (i.e., a Seifert fiber space of type $S^{2}(2,2, x)$ for some $\left.x \geq 2\right)$.

Proof. First we recall the diagram below (see the proof of Lemma 4.3), where the slope $\gamma$ corresponds with the integer $m$.

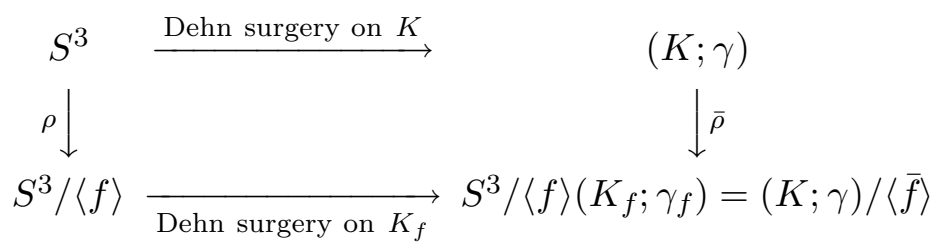

Since the meridional slope $\mu_{f}$ of $K_{f}$ is a cyclic surgery slope and $\gamma_{f}$ is a finite surgery slope of $K_{f}$, [6, Theorem 1.1 (2)], together with the argument in the proof of Lemma 4.3, shows that the period $p$ is 2 .

Thus $\bar{f}$ is a free involution on $(K ; \gamma)$, which is a Seifert fiber space of type $S^{2}(2,2, x)(x \geq 1)$ excluding $S^{2} \times S^{1}$. We can then apply [44, Theorem 8] to conclude that $(K ; \gamma) /\langle\bar{f}\rangle$ is a lens space $\left(¥ S^{2} \times S^{1}\right)$ or a prism manifold.

$\square$ (Claim 4.9)

If $(K ; \gamma) /\langle\bar{f}\rangle$ is a lens space, then the cyclic surgery theorem [11] shows that the period $p=1$, a contradiction. Assume that $(K ; \gamma) /\langle\bar{f}\rangle$ is a prism 
manifold. Then its fundamental group is a D-type finite group in $[6]([44$, p.131]). Applying [6, Thoerm 1.5 (1)] to the cyclic surgery slope $\mu_{f}$ and the D-type finite surgery slope $\gamma_{f}$ of $K_{f}$, we see that their distance, which coincides with the period $p$, must be 1 , a contradiction. Thus $(K ; m)$ cannot be a Seifert fiber space over $\mathbb{R} P^{2}$ with at most one exceptional fiber.

This finishes the proof of Theorem 4.5.

$\square$ (Theorem 4.5)

\subsection{Surgeries on knots with Type F symmetry.}

In this subsection we prove:

Theorem 4.10. Let $K$ be a hyperbolic, freely periodic knot with period $p>2$. Then $(K ; r)$ cannot be a toroidal Seifert fiber space.

Before proving the theorem, we observe the following. Recall from Theorem 4.5 that the base surface of $(K ; r)$ is the 2 -sphere.

Lemma 4.11. If $(K ; r)$ is a small Seifert fiber space which contains an incompressible torus, then $K$ is a trefoil knot and $r=0$.

Proof. Let us assume that $(K ; r)$ has at most three exceptional fibers and contains an incompressible torus $T$. Then $(K ; r)$ has exactly three exceptional fibers and $T$ is non-separating ([27, VI.13]). Hence $r=0$ and $(K ; r)$ is a torus bundle over $S^{1}$. From [17, Corollary 8.23], we see that $K$ is a trefoil knot or the figure-eight knot. However 0-surgery on the figure-eight knot does not yield a Seifert fiber space, hence $K$ is a trefoil knot and $r=0$.

$\square$ (Lemma 4.11)

It follows that if $(K ; r)$ is a toroidal Seifert fiber space over $S^{2}$ for a hyperbolic knot $K$, then it contains more than three exceptional fibers, equivalently the base orbifold contains more than three cone points.

Proof of Theorem 4.10. Let $f$ be a diffeomorphism of $S^{3}$ giving the free period $p(>2)$ of $K$, and that $\bar{f}$ is a diffeomorphism of $(K ; r)$ of period $p$ induced from $f$. Suppose for a contradiction that $(K ; r)$ is a toroidal Seifert fiber space. By Lemma 3.1 (1) we have a Seifert fibration which is invariant under $\langle\bar{f}\rangle$, and $\bar{f}$ induces an isomorphism $\hat{f}$ on the base orbifold $B$ $\left(|B|=S^{2}\right)$. There are four cases to consider (see Section 3):

(1) $\hat{f}: S^{2} \rightarrow S^{2}$ is the identity map,

(2) $\hat{f}: S^{2} \rightarrow S^{2}$ is nontrivial rotation, 
(3) $\hat{f}: S^{2} \rightarrow S^{2}$ is a reflection, or

(4) $\hat{f}: S^{2} \rightarrow S^{2}$ is orientation reversing and $\operatorname{Fix}(\hat{f})=\emptyset$.

Case (1) : $\hat{f}$ is the identity map. By taking some power of $f$, we may assume that $f^{x}$ has a prime period $p^{\prime} \geq 2$, so that $\operatorname{Fix}\left(\overline{f^{x}}\right)=K^{*}$ or $\operatorname{Fix}\left(\left(\overline{f^{x}}\right)^{i}\right)=\emptyset$ for $1 \leq i \leq p^{\prime}-1$. Since $\hat{f}=$ id., $\widehat{f}^{x}=\hat{f}^{x}=\mathrm{id}$.. If $\operatorname{Fix}\left(\overline{f^{x}}\right)=K^{*}$, then by Lemma $4.1 K$ is a torus knot, a contradiction. If $\operatorname{Fix}\left(\left(\overline{f^{x}}\right)^{i}\right)=\emptyset$ for $1 \leq i \leq p^{\prime}-1$, then since $\widehat{f^{x}}=\mathrm{id}$., there exists an $\widehat{f^{x}}$-essential loop on $B$. This contradicts Lemma 4.3.

Case (2) : $\hat{f}$ is a nontrivial rotation. The base orbifold $B$ contains more than three cone points (Lemma 4.11), while $\operatorname{Fix}(\hat{f})$ consists of two points. So there is a cone point $z$ which is not belonging to $\operatorname{Fix}(\hat{f})$. If the period of $\hat{f}$ is greater than 2 , then there are three cone points of the same index. This is impossible by the following lemma.

Lemma 4.12. Assume that $(K ; r)$ is a Seifert fiber space over $S^{2}$. Then the base orbifold $B$ contains at most two cone points of the same index.

Proof. Suppose that $(K ; r)$ is a Seifert fiber space of type $S^{2}\left(\alpha_{1}, \alpha_{2}, \alpha_{3}, \ldots, \alpha_{k}\right)$. Then the fundamental group $\pi_{1}((K ; r))$ has a presentation $([27])$

$$
<c_{1}, c_{2}, c_{3}, \ldots, c_{k}, h \mid c_{i} h c_{i}^{-1} h^{-1}=1, c_{i}^{\alpha_{i}}=h^{\beta_{i}}, h^{b}=c_{1} c_{2} c_{3} \cdots c_{k}>
$$

for some integers $\beta_{i}$ and $b$. Adding the relation $h=1$ and abelianizing, we have a presentation

$$
<c_{1}, c_{2}, c_{3}, \ldots, c_{k} \mid \alpha_{i} c_{i}=0, c_{1}+c_{2}+c_{3}+\cdots+c_{k}=0>.
$$

Now assume for a contradiction, without loss of generality, that $\alpha_{1}=\alpha_{2}=$ $\alpha_{3}=\alpha \geq 2$. Then adding further relations $c_{4}=\cdots=c_{k}=0$, we have $<c_{1}, c_{2}, c_{3}\left|\alpha c_{i}=0, c_{1}+c_{2}+c_{3}=0>\cong<c_{1}, c_{2}\right| \alpha c_{1}=\alpha c_{2}=0>\cong \mathbb{Z}_{\alpha} \oplus \mathbb{Z}_{\alpha}$. This contradicts that $H_{1}((K ; r))$ being cyclic. Thus $(K ; r)$ contains at most three exceptional fibers of the same index and the result follows. $\square$ (Lemma 4.12)

Henceforth $\hat{f}$ is a nontrivial rotation of order 2. Then since $\bar{f}^{p}=$ id., we have $\hat{f}^{p}=\mathrm{id}$. and hence $p$ is even. Consider $f^{x}$ for some $x$ with $x \mid p$ so that $f^{x}$ has a prime period $p^{\prime}$ and $\operatorname{Fix}\left(\overline{f^{x}}\right)=K^{*}$ or $\operatorname{Fix}\left(\left(\overline{f^{x}}\right)^{i}\right)=\emptyset$ for $1 \leq i \leq p^{\prime}-1$. We note here that $x>1$; for otherwise, the period $p$ of 
$f=f^{x}$ is even prime, hence $p=2$, contradicting the assumption. Now we divide into two cases depending on whether $x$ is even or odd. If $x$ is even, then since $\hat{f}^{2}=$ id., $\widehat{f}^{x}=\hat{f}^{x}=$ id.. Let us apply the argument in Case (1) to $f^{x}$ to get a contradiction. If $x$ is odd, then since $x>1$ and $x \mid p$, the period $p$ is not of the form $2^{n}$. Hence we can choose $f^{y}$ so that $f^{y}$ has an odd prime period $q \geq 3$; since the period is prime, we have $\operatorname{Fix}\left(\overline{f^{y}}\right)=K^{*}$ or $\operatorname{Fix}\left(\left(\overline{f^{y}}\right)^{i}\right)=\emptyset$ for $1 \leq i \leq q-1$ for $f^{y}$.

Claim 4.13. $\widehat{f^{y}}: S^{2} \rightarrow S^{2}$ is the identity map.

Proof. Since $\left(\overline{f^{y}}\right)^{q}=\mathrm{id}$., we have $\left(\widehat{f^{y}}\right)^{q}=\mathrm{id}$.. On the other hand, $\left(\widehat{f^{y}}\right)^{q}=$ $\left(\hat{f}^{q}\right)^{y}=\hat{f}^{y}=\widehat{f^{y}}$, because $\hat{f}^{2}=\mathrm{id}$. and $q$ is odd. Thus $\widehat{f^{y}}=\left(\widehat{f^{y}}\right)^{q}=\mathrm{id}$..

$\square($ Claim 4.13)

From Lemma 4.1, we have the only possibility: $\operatorname{Fix}\left(\left(\overline{f^{y}}\right)^{i}\right)=\emptyset$ for $1 \leq$ $i \leq q-1$. Then Claim 4.13 assures an existence of an $\widehat{f^{y}}$-essential loop on B. This contradicts Lemma 4.3 .

Case $(3): \hat{f}$ is a reflection. Let $x$ be a point in $\operatorname{Fix}(\hat{f})$. Then $\bar{f}$ induces an action on the fiber $t=\pi^{-1}(x)$. Since $\hat{f}$ is a reflection, $\left.\bar{f}\right|_{t}$ reverses an orientation of $t$. This implies that $\bar{f}$ has a nonempty fixed point set. Thus $\operatorname{Fix}(\bar{f})=K^{*}$. Then since $K$ is not a torus knot, from Lemma 4.2, we see that the period $p=2$, contradicting the assumption.

Case (4) $: \hat{f}$ is orientation reversing and $\operatorname{Fix}(\hat{f})=\emptyset$. First suppose that the period of $\hat{f}$ is greater than 2 . Then we have the following, which contradicts Lemma 4.12.

Claim 4.14. There exist three cone points of the same index.

Proof. Since $\hat{f}^{2}$ is orientation preserving and $\hat{f}^{2} \neq \mathrm{id}$., $\operatorname{Fix}\left(\hat{f}^{2}\right)$ consists of two points. On the other hand, there are more than three cone points on $S^{2}$ (Lemma 4.11), so we can take a cone point $z \in S^{2}-\operatorname{Fix}\left(\hat{f}^{2}\right)$. Then it is easy to see that $z, \hat{f}(z)$ and $\hat{f}^{2}(z)$ are distinct from each other and they have the same index.

$\square($ Claim 4.14)

Therefore $\hat{f}$ has period 2, i.e., $\hat{f}$ is an antipodal map. Let us consider $f^{x}$ which has a prime period $p^{\prime} \geq 2$ so that $\operatorname{Fix}\left(\overline{f^{x}}\right)=K^{*}$ or $\operatorname{Fix}\left(\left(\overline{f^{x}}\right)^{i}\right)=\emptyset$ for $1 \leq i \leq p^{\prime}-1$. Note that $\widehat{f^{x}}=\hat{f}^{x}=$ id. (if $x$ is even) or $\widehat{f^{x}}=\hat{f}^{x}=\hat{f}$ (if $x$ is odd).

First suppose that $\operatorname{Fix}\left(\overline{f^{x}}\right)=K^{*}$. Then $\operatorname{Fix}\left(\widehat{f^{x}}\right) \neq \emptyset$, thus $\widehat{f^{x}} \neq \hat{f}$, and hence $\widehat{f^{x}}=\mathrm{id}$.. From Lemma 4.1, we see that $K$ is a torus knot, contradicting the assumption. 
Next suppose that $\operatorname{Fix}\left(\left(\overline{f^{x}}\right)^{i}\right)=\emptyset$ for $1 \leq i \leq p^{\prime}-1$. In either case $\widehat{f^{x}}=$ id. or $\widehat{f^{x}}=\hat{f}$, we can find an $\widehat{f^{x}}$-essential loop $c$ so that $\widehat{f^{x}}(c)=c$ or $\widehat{f^{x}}(c) \cap c=\emptyset$, respectively. This then contradicts Lemma 4.3.

The proof of Theorem 4.10 is now completed.

$\square$ (Theorem 4.10)

\subsection{Surgeries on knots with Type SF symmetry.}

We are ready to prove:

Theorem 4.15. Let $K$ be a hyperbolic knot with a semi-free period $p>2$. Then $(K ; r)$ cannot be a toroidal Seifert fiber space.

Proof. Let $f:\left(S^{3}, K\right) \rightarrow\left(S^{3}, K\right)$ be an orientation preserving periodic diffeomorphism such that $\operatorname{Fix}(f)=\emptyset$ but $\operatorname{Fix}\left(f^{i}\right) \neq \emptyset$ for some $i(1<i<p)$. Suppose for a contradiction that $(K ; r)$ is a toroidal Seifert fiber space, and choose a Seifert fibration of $(K ; r)$ which is invariant under $\langle\bar{f}\rangle$ (Lemma 3.1 $(1))$.

Note that $f^{i}$ gives a cyclic period of $K$. From [36, Theorem 1.3], we see that $(K ; r)$ cannot be a Seifert fiber space over $\mathbb{R} P^{2}$. Thus in the following, we assume that the base surface $|B|$ is $S^{2}$.

Since $f^{i}$ gives a cyclic period of the hyperbolic knot $K$, we have:

Claim 4.16. $\widehat{f}^{i}: S^{2} \rightarrow S^{2}$ reverses an orientation of $S^{2}$.

Proof. Recall from [35, Proposition 5.1] that a circle $L=\operatorname{Fix}\left(f^{i}\right) \subset S^{3}$ cannot become a fiber in $(K ; r)$. (We can regard $L \subset(K ; r)$, because $L \subset E(K)$.) Since $L \subset \operatorname{Fix}\left(\overline{f^{i}}\right)$, Lemma 3.2(1) shows that $\widehat{f}^{i}$ reverses an orientation of $|B|=S^{2}$.

$\square($ Claim 4.16)

It follows from Claim 4.16 that $\hat{f}$ reverses an orientation of $S^{2}$ and $i$ is an odd integer. We note that since $i>1$, we have $i \geq 3$.

Claim 4.17. $f^{2}:\left(S^{3}, K\right) \rightarrow\left(S^{3}, K\right)$ gives a free period of $K$ with period $i$ which is greater than 2 .

Proof. First we show that $f^{2}$ has period $i \geq 3$. By Theorem 2.4, the periodic diffeomorphism $f^{i}$ has period 2. Hence $f^{2 i}=\left(f^{i}\right)^{2}=$ id., so we have $2 i=p k$ for some integer $k \geq 1$. Since $i<p$, we have $k=1$, i.e., $p=2 i$. This means that $f^{2}$ has period $i \geq 3$.

Next we show that $\operatorname{Fix}\left(\left(f^{2}\right)^{j}\right)=\emptyset$ for $1 \leq j \leq i-1$. Suppose for a contradiction that $\operatorname{Fix}\left(\left(f^{2}\right)^{x}\right) \neq \emptyset$ for some $x$ such that $1 \leq x \leq i-1$. Then 
$f^{2 x}=\left(f^{2}\right)^{x}$ gives a cyclic period of $K$, and again by Theorem 2.4 , the period of $f^{2 x}$ is 2 , and hence, $\left(f^{2}\right)^{2 x}=\left(f^{2 x}\right)^{2}=\mathrm{id}$.. On the other hand, since $f^{2}$ has period $i$, we have $i \mid 2 x$, which also implies $i \mid x$, because $i(\geq 3)$ is odd. This contradicts the assumption $1 \leq x \leq i-1$. It follows that $f^{2}$ gives a free period of $K$ with period $i \geq 3$.

$\square($ Claim 4.17)

Then Claim 4.17 contradicts Theorem 4.10. $\square$ (Theorem 4.15)

\section{Strong inversions and Seifert fibered surgeries.}

In this section we study Seifert fibered surgeries on strongly invertible knots.

Let $g: S^{3} \rightarrow S^{3}$ be a strong inversion of $K$ with $\operatorname{Fix}(g)=L_{g}$ and let $N(K)$ be a $\langle g\rangle$-invariant tubular neighborhood of $K$ in $S^{3}$. Then we can extend $\left.g\right|_{S^{3}-\operatorname{int} N(K)}$ over $(K ; r)$ to get an involution $\bar{g}:(K ; r) \rightarrow(K ; r)$. Since $L_{g}$ intersects $K$ in two points, $L_{g} \cap E(K)$ consists of two properly embedded $\operatorname{arcs} c_{1}$ and $c_{2}$ in $E(K)$. On the other hand, $\bar{g}$ fixes two properly embedded $\operatorname{arcs} c_{1}^{\prime}$ and $c_{2}^{\prime}$ in the filling solid torus; each $c_{i}^{\prime}(i=1,2)$ intersects $K^{*}$ (the core of the filling solid torus) in a single point. Thus the fixed point set $\operatorname{Fix}(\bar{g})$ consists of a single circle $L_{g}^{\prime}$ or two circles $L_{g}^{\prime} \cup L_{g}^{\prime \prime}$ depending on how $c_{i}(i=1,2)$ and $c_{j}^{\prime}(j=1,2)$ are connected in $(K ; r)$.

In what follows we assume that $(K ; r)$ is a Seifert fiber space over $S^{2}$. By Lemma 3.1 we choose a Seifert fibration of $(K ; r)$ which is preserved by $\bar{g}$ so that we can obtain an isomorphism $\hat{g}$ on the base orbifold $B$.

Here we introduce,

Condition $(\hat{g}) \quad$ The induced automorphism $\hat{g}$ of the base surface $|B|=S^{2}$ is orientation preserving.

If we have Condition $(\hat{g})$, then by Lemma $3.2, \operatorname{Fix}(\bar{g})$ consists of fibers in $(K ; r)$. The goal in this section is proving the following result.

Proposition 5.1. Assume that Condition ( $\hat{g})$ holds.

(1) If $(K ; r)$ contains more than three exceptional fibers, then $\operatorname{Fix}(\bar{g})$ consists of exactly two components $L_{g}^{\prime}$ and $L_{g}^{\prime \prime}$, and both $L_{g}^{\prime}$ and $L_{g}^{\prime \prime}$ are regular fibers in $(K ; r)$.

(2) If $(K ; r)$ is a small Seifert fiber space such that indices of exceptional fibers are distinct from each other, then a component of $\operatorname{Fix}(\bar{g})$ is an exceptional fiber of index 2.

Let $\bar{\psi}:(K ; r) \rightarrow(K ; r) /\langle\bar{g}\rangle$ be a 2 -fold cyclic branched covering induced from the action of $\langle\bar{g}\rangle$ on $(K ; r)$. 
Lemma 5.2. The quotient space $(K ; r) /\langle\bar{g}\rangle$ is diffeomorphic to $S^{3}$, and $\bar{\psi}$ branches along (possibly exceptional) fibers in some Seifert fibration of $S^{3}$ and preserves Seifert fibrations.

Proof. The Montesinos trick [38] ([1]) shows that the quotient space $(K ; r) /\langle\bar{g}\rangle$ can be obtained from $S^{3}=S^{3} /\langle g\rangle$ by performing an untangle surgery, and hence $(K ; r) /\langle\bar{g}\rangle$ is also the 3 -sphere (diagram below).

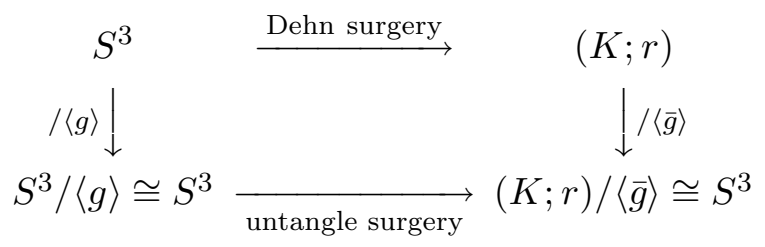

Moreover since $\bar{g}$ preserves the Seifert fibration of $(K ; r)$ and Fix $(\bar{g})$ consists of fibers (Condition $(\hat{g})$ and Lemma 3.2), the image of each fiber in $(K ; r)$ by $\bar{\psi}$ is a circle, so that $S^{3} \cong(K ; r) /\langle\bar{g}\rangle$ is a union of these pairwise disjoint circles $\mathcal{F}$. Then $\mathcal{F}$ defines a Seifert fibration of $S^{3} \cong(K ; r) /\langle\bar{g}\rangle$. In the following we give a sketch of the proof of this fact.

Let $\tilde{t}$ be a fiber in $(K ; r)$ and $N(\tilde{t})$ a $\langle\bar{g}\rangle$-equivariant fibered tubular neighborhood of $\tilde{t}$. If $\bar{g}(\tilde{t}) \neq \tilde{t}$, then $(N(\tilde{t}) \cup \bar{g}(N(\tilde{t}))) /\langle\bar{g}\rangle$ is the required fibered neighborhood of $t=\bar{\psi}(\tilde{t})$. Suppose that $\bar{g}(\tilde{t})=\tilde{t}$; then we have also $\bar{g}(N(\tilde{t}))=N(\tilde{t})$. It should be noted here that since Condition $(\hat{g})$ holds, $\left.\bar{g}\right|_{\tilde{t}}$ preserves an orientation of $\tilde{t}$. Let us take a finite cyclic covering $S^{1} \times D^{2}$ of $N(\tilde{t})$ so that the preimage of each fiber in $N(\tilde{t})$ is $S^{1} \times\{x\} \subset S^{1} \times D^{2}$ for some $x \in D^{2}$. Let $\Gamma$ be the group of all the diffeomorphisms of $S^{1} \times D^{2}$ which lift $\langle\bar{g}\rangle$. Clearly $\Gamma$ is a finite group and $\left(S^{1} \times D^{2}\right) / \Gamma=N(\tilde{t}) /\langle\bar{g}\rangle$. Furthermore since $\langle\bar{g}\rangle$ preserves the Seifert fibration of $N(\tilde{t}), \Gamma$ also preserves the Seifert fibration of $S^{1} \times D^{2}$. Let $D$ be a $\Gamma$-equivariant meridian disk of $S^{1} \times D^{2}([32])$; we may assume that $D$ intersects each fiber $S^{1} \times\{x\}$ transversely. Then by taking a conjugation by a diffeomorphism of the form id. $\times \eta$ of $S^{1} \times D^{2}$, we may assume that the induced action of $\Gamma$ on the second factor $D^{2}$ is a finite cyclic action generated by a standard rotation at the center $(0,0) \in D^{2}$. Then it turns out that $N(\tilde{t}) /\langle\bar{g}\rangle=\left(S^{1} \times D^{2}\right) / \Gamma$ is the required fibered neighborhood of $t=\bar{\psi}(\tilde{t})$ such that $\left.\bar{\psi}\right|_{N(\tilde{t})}: N(\tilde{t}) \rightarrow N(\tilde{t}) /\langle\bar{g}\rangle$ preserves Seifert fibrations.

$\square$ (Lemma 5.2)

The next lemma describes a relation between indices of fibers $\tilde{t}$ and $\bar{\psi}(\tilde{t})$.

Lemma 5.3. Let $\tilde{t}$ be a fiber in $(K ; r)$ which covers a fiber $t$ in $(K ; r) /\langle\bar{g}\rangle \cong$ $S^{3}$. Then we have: 
(1) If $\bar{g}(\tilde{t}) \neq \tilde{t}$, then index $(\tilde{t})=\operatorname{index}(t)$.

(2) If $\bar{g}(\tilde{t})=\tilde{t}$ and $\left.\bar{g}\right|_{\tilde{t}}: \tilde{t} \rightarrow \tilde{t}$ is a rotation, then $\operatorname{index}(\tilde{t})=\operatorname{index}(t)$ or $\frac{\text { index }(t)}{2}$

(3) If $\bar{g}(\tilde{t})=\tilde{t}$ and $\left.\bar{g}\right|_{\tilde{t}}: \tilde{t} \rightarrow \tilde{t}$ is the identity map, then $\operatorname{index}(\tilde{t})=\operatorname{index}(t)$ or $2 \operatorname{index}(t)$.

Proof. If (1) happens, then $(N(\tilde{t}) \cup \bar{g}(N(\tilde{t}))) /\langle\bar{g}\rangle=N(\tilde{t})$ gives a fibered neighborhood $N(t)$ of $t$ in $(K ; r) /\langle\bar{g}\rangle$. Hence index $(\tilde{t})=\operatorname{index}(t)$.

To prove (2) and (3), let $N(\tilde{t})$ be a $\langle\bar{g}\rangle$-invariant fibered tubular neighborhood of $\tilde{t}$ in $(K ; r)$. Then we have $\left.\bar{\psi}\right|_{N(\tilde{t})}: N(\tilde{t}) \rightarrow N(\tilde{t}) /\langle\bar{g}\rangle=N(t)$, which is fiber preserving. Let $\tilde{\mu}, \tilde{\lambda}$ (resp. $\mu, \lambda$ ) be a meridian-longitude pair of $N(\tilde{t})$ (resp. $N(t))$. A regular fiber on $\partial N(\tilde{t})$ represents $p \tilde{\mu}+q \tilde{\lambda}$ for some relatively prime integers $p$ and $q(\geq 1)$, where $q$ is the index of $\tilde{t}$. (Note that $\tilde{t}$ is a regular fiber if and only if $q=1$. )

If (2) happens, then $\bar{\psi}(p \tilde{\mu}+q \tilde{\lambda})=p \mu+q(2 \lambda+x \mu)=(p+q x) \mu+2 q \lambda=$ $(p+q x, 2 q)\left\{\frac{p+q x}{(p+q x, 2 q)} \mu+\frac{2 q}{(p+q x, 2 q)} \lambda\right\}$ for some $x$. Since $p$ and $q$ are relatively prime, $(p+q x, 2 q)=1$ or 2 , and hence the $\operatorname{index}(t)=\frac{2 q}{(p+q x, 2 q)}$ is $2 q$ or $q$.

If (3) occurs, then we have

$$
\begin{aligned}
\bar{\psi}(p \tilde{\mu}+q \tilde{\lambda}) & =2 p \mu+q(\lambda+x \mu) \\
& =(2 p+q x) \mu+q \lambda \\
& =(2 p+q x, q)\left\{\frac{2 p+q x}{(2 p+q x, q)} \mu+\frac{q}{(2 p+q x, q)} \lambda\right\}
\end{aligned}
$$

for some $x$. Since $p$ and $q$ are relatively prime, $(2 p+q x, q)=(2, q)$. Hence the $\operatorname{index}(t)=\frac{q}{(2 p+q x, q)}=\frac{q}{(2, q)}=q$ or $\frac{q}{2}$ depending on whether $q$ is odd or even.

$\square$ (Lemma 5.3)

In particular, we have:

Lemma 5.4. Let $t$ be a regular fiber in $S^{3}=(K ; r) /\langle\bar{g}\rangle$ and $\tilde{t}$ a fiber in $(K ; r)$ such that $\bar{\psi}(\tilde{t})=t$. Then the index of $\tilde{t}$ is at most two; if the index is two, then $t$ is a component of $\operatorname{Fix}(\bar{g}) /\langle\bar{g}\rangle$ and $\bar{\psi}^{-1}\left(t^{\prime}\right)$ is connected for a regular fiber $t^{\prime}$ near $t$.

Proof. Since $t$ is a regular fiber, i.e., $\operatorname{index}(t)=1$, the first assertion follows directly from Lemma 5.3. Furthermore, $\tilde{t}$ can be an exceptional fiber only when we have (3) with $q=2$ in the above lemma. This means that $\tilde{t}$ is a component of $\operatorname{Fix}(\bar{g})$ and $t=\bar{\psi}(\tilde{t})$ is a component of $\operatorname{Fix}(\bar{g}) /\langle\bar{g}\rangle$. To observe 
the last assertion, let $\tilde{t^{\prime}}(\subset \partial N(\tilde{t}))$ be a regular fiber representing $p \tilde{\mu}+2 \tilde{\lambda}$ which covers a regular fiber $t^{\prime}(\subset \partial N(t))$. The argument in the proof of Lemma 5.3 shows that $\bar{\psi}(p \tilde{\mu}+2 \tilde{\lambda})=2\{(p+x) \mu+\lambda\}$. Hence the regular fiber $\tilde{t}^{\prime}$ doubly covers the regular fiber $t^{\prime}$, and thus $\bar{\psi}^{-1}\left(t^{\prime}\right)$ is connected.

$\square$ (Lemma 5.4)

Proof of Proposition 5.1. As in the previous lemmas, let $\bar{\psi}:(K ; r) \rightarrow$ $(K ; r) /\langle\bar{g}\rangle=S^{3}$ be the branched covering projection, and let $\psi$ be a restriction of $\bar{\psi}$ to $(K ; r)-\operatorname{Fix}(\bar{g})$, so that $\psi$ is an unbranched covering projection.

Since $S^{3}$ has a Seifert fibration over $S^{2}$ (with at most two exceptional fibers), the base surface of $X=(K ; r) /\langle\bar{g}\rangle-\operatorname{Fix}(\bar{g}) /\langle\bar{g}\rangle$ is a once or twice punctured sphere depending on whether $\operatorname{Fix}(\bar{g})=L_{g}^{\prime}$ or $L_{g}^{\prime} \cup L_{g}^{\prime \prime}$. If $\operatorname{Fix}(\bar{g})=$ $L_{g}^{\prime}$, then $X$ contains at least two exceptional fibers, and if $\operatorname{Fix}(\bar{g})=L_{g}^{\prime} \cup L_{g}^{\prime \prime}$, then $X$ contains at least one exceptional fiber, for otherwise $(K ; r)$ would be a union of two solid tori, i.e., a lens space (possibly $S^{3}$ or $S^{2} \times S^{1}$ ), a contradiction.

Let us prove Proposition 5.1 (1). First assume that $\operatorname{Fix}(\bar{g})=L_{g}^{\prime}$. Then from Lemma 5.2 , we see that $(K ; r)$ is a 2 -fold cyclic branched cover of $S^{3}$ branched along the (nontrivial) torus knot $L_{g}^{\prime} /\langle\bar{g}\rangle$, which is a regular fiber. Let $c_{1}, c_{2}$ be exceptional fibers of indices $p, q$ in $(K ; r) /\langle\bar{g}\rangle \cong S^{3} ; p$ and $q$ are relatively prime.

Here we recall the following from covering space theory.

Claim 5.5. Let $c$ be a knot in $X=(K ; r) /\langle\bar{g}\rangle-L_{g}^{\prime} /\langle\bar{g}\rangle$. Then $c$ can be lifted to $(K ; r)-L_{g}^{\prime}\left(\right.$ i.e., $\psi^{-1}(c)$ consists of two components) if and only if the linking number $\ell k\left(c, L_{g}^{\prime} /\langle\bar{g}\rangle\right)$ is even.

Hence if $(K ; r)$ has more than three exceptional fibers, then by Lemma 5.4 there are two possibilities:

(i) $L_{g}^{\prime}$ is an exceptional fiber and at least one of $\psi^{-1}\left(c_{1}\right)$ and $\psi^{-1}\left(c_{2}\right)$ consists of two components, or

(ii) $L_{g}^{\prime}$ is a regular fiber and both $\psi^{-1}\left(c_{1}\right)$ and $\psi^{-1}\left(c_{2}\right)$ consist of two components.

If (i) happens, then by Lemma $5.4, \psi^{-1}\left(t^{\prime}\right)$ is connected for a regular fiber $t^{\prime}$ near $L_{g}^{\prime} /\langle\bar{g}\rangle$. This implies that $\ell k\left(t^{\prime}, L_{g}^{\prime} /\langle\bar{g}\rangle\right)=p q$ is odd by Claim 5.5 , hence both $p$ and $q$ are odd integers. It then follows from Claim 5.5, $\psi^{-1}\left(c_{i}\right)$ consists of only one component for $i=1,2$, a contradiction.

If (ii) happens, then both $\ell k\left(c_{1}, L_{g}^{\prime} /\langle\bar{g}\rangle\right)=q$ and $\ell k\left(c_{2}, L_{g}^{\prime} /\langle\bar{g}\rangle\right)=p$ are even integers by Lemma 5.5, which contradicts the fact that $p$ and $q$ are relatively prime. 
Next we consider the case where $\operatorname{Fix}(\bar{g})=L_{g}^{\prime} \cup L_{g}^{\prime \prime}$. Recall that $S^{3}=(K ; r) /\langle\bar{g}\rangle$ has at most two exceptional fibers, while $X=(K ; r) /\langle\bar{g}\rangle-$ $\left(L_{g}^{\prime} /\langle\bar{g}\rangle \cup L_{g}^{\prime \prime} /\langle\bar{g}\rangle\right)$ contains at least one exceptional fiber in $S^{3}=(K ; r) /\langle\bar{g}\rangle$ (see the second paragraph of the proof of Proposition 5.1). Thus we have the following two possibilities:

(i) $X$ has one exceptional fiber $c_{1}$; in this case at least one of $L_{g}^{\prime} /\langle\bar{g}\rangle$ and $L_{g}^{\prime \prime} /\langle\bar{g}\rangle$ is a regular fiber, or

(ii) $X$ has two exceptional fibers $c_{1}$ and $c_{2}$; in this case both $L_{g}^{\prime} /\langle\bar{g}\rangle$ and $L_{g}^{\prime \prime} /\langle\bar{g}\rangle$ are regular fibers.

We recall again the following from covering space theory.

Claim 5.6. Let $c$ be a knot in $X=(K ; r) /\langle\bar{g}\rangle-\left(L_{g}^{\prime} /\langle\bar{g}\rangle \cup L_{g}^{\prime \prime} /\langle\bar{g}\rangle\right)$. Then $c$ can be lifted to $(K ; r)-\left(L_{g}^{\prime} \cup L_{g}^{\prime \prime}\right)$ (i.e., $\psi^{-1}(c)$ consists of two components) if and only if $\ell k\left(c, L_{g}^{\prime} /\langle\bar{g}\rangle\right)+\ell k\left(c, L_{g}^{\prime \prime} /\langle\bar{g}\rangle\right)$ is even.

Suppose that (i) happens. Without loss of generality, we assume that $L_{g}^{\prime} /\langle\bar{g}\rangle$ is a regular fiber.

Since $(K ; r)$ contains more than three exceptional fibers and any fiber in $(K ; r)$ which covers a regular fiber in $X$ is also a regular fiber (Lemma 5.4 ), both $L_{g}^{\prime}$ and $L_{g}^{\prime \prime}$ are exceptional fibers and $\psi^{-1}\left(c_{1}\right)$ consists of two components.

Let $c$ be a fiber in $S^{3}=(K ; r) /\langle\bar{g}\rangle$ which is a core of a solid torus $S^{3}-\operatorname{int} N\left(c_{1}\right) ; c$ is possibly a regular fiber. Note that if $S^{3}-\operatorname{int} N\left(c_{1}\right)$ is exceptionally fibered, then necessarily $c \neq L_{g}^{\prime} /\langle\bar{g}\rangle$, and if $S^{3}-\operatorname{int} N\left(c_{1}\right)$ is regularly fibered, then we choose $c$ so that $c \neq L_{g}^{\prime} /\langle\bar{g}\rangle$. We now consider two subcases: $L_{g}^{\prime \prime} /\langle\bar{g}\rangle=c$ or $L_{g}^{\prime \prime} /\langle\bar{g}\rangle \neq c$.

First suppose that $L_{g}^{\prime \prime} /\langle\bar{g}\rangle=c$; then $\ell k\left(c_{1}, L_{g}^{\prime \prime} /\langle\bar{g}\rangle\right)=\ell k\left(c_{1}, c\right)=1$, $\ell k\left(L_{g}^{\prime} /\langle\bar{g}\rangle, L_{g}^{\prime \prime} /\langle\bar{g}\rangle\right)=\ell k\left(L_{g}^{\prime} /\langle\bar{g}\rangle, c\right)=q$ for some integer $q(\geq 2)$, which coincides with the index of $c_{1}$. Finally we put $\ell k\left(c_{1}, L_{g}^{\prime} /\langle\bar{g}\rangle\right)=p(\geq 1)$, which coincides with the index of $L_{g}^{\prime \prime} /\langle\bar{g}\rangle=c$. Since $L_{g}^{\prime}$ is an exceptional fiber, by Lemma $5.4 \psi^{-1}\left(t^{\prime}\right)$ is connected for a regular fiber $t^{\prime}$ near $L_{g}^{\prime} /\langle\bar{g}\rangle$. Thus $\ell k\left(t^{\prime}, L_{g}^{\prime} /\langle\bar{g}\rangle\right)+\ell k\left(t^{\prime}, L_{g}^{\prime \prime} /\langle\bar{g}\rangle\right)=p q+q=(p+1) q$ is odd (Claim 5.6). On the other hand, since $\psi^{-1}\left(c_{1}\right)$ has two components, $\ell k\left(c_{1}, L_{g}^{\prime} /\langle\bar{g}\rangle\right)+\ell k\left(c_{1}, L_{g}^{\prime \prime} /\langle\bar{g}\rangle\right)=p+1$ is even (Claim 5.6). This is a contradiction.

Next suppose that $L_{g}^{\prime \prime} /\langle\bar{g}\rangle \neq c$. Then $c$ is contained in $X$ and hence it is a regular fiber. We note that since fibers other than $c$ and $c_{1}$ are regular fibers in $S^{3}=(K ; r) /\langle\bar{g}\rangle, L_{g}^{\prime \prime} /\langle\bar{g}\rangle$ is also a regular fiber. It follows that $\ell k\left(c_{1}, L_{g}^{\prime} /\langle\bar{g}\rangle\right)=\ell k\left(c_{1}, L_{g}^{\prime \prime} /\langle\bar{g}\rangle\right)=1$, which coincides with the index of $c$. We have $\ell k\left(c, L_{g}^{\prime} /\langle\bar{g}\rangle\right)=\ell k\left(c, L_{g}^{\prime \prime} /\langle\bar{g}\rangle\right)=q(\geq 2)$, which coincides with the index 
of $c_{1}$. Since $L_{g}^{\prime}$ is an exceptional fiber, by Lemma $5.4 \psi^{-1}\left(t^{\prime}\right)$ is connected for a regular fiber $t^{\prime}$ near $L_{g}^{\prime} /\langle\bar{g}\rangle$. Thus $\ell k\left(t^{\prime}, L_{g}^{\prime} /\langle\bar{g}\rangle\right)+\ell k\left(t^{\prime}, L_{g}^{\prime \prime} /\langle\bar{g}\rangle\right)=q+q=2 q$ would be odd (Claim 5.6), a contradiction.

Let us assume that (ii) happens. We put $\ell k\left(c_{1}, L_{g}^{\prime} /\langle\bar{g}\rangle\right)=p$ and $\ell k\left(c_{2}, L_{g}^{\prime} /\langle\bar{g}\rangle\right)=q$. Then $p$ coincides with the index of $c_{2}$ and $q$ coincides with that of $c_{1}$; we have also $\ell k\left(c_{1}, L_{g}^{\prime \prime} /\langle\bar{g}\rangle\right)=p$ and $\ell k\left(c_{2}, L_{g}^{\prime \prime} /\langle\bar{g}\rangle\right)=q$. Let $t^{\prime}$ be a regular fiber near $L_{g}^{\prime} /\langle\bar{g}\rangle$. Since $\ell k\left(t^{\prime}, L_{g}^{\prime} /\langle\bar{g}\rangle\right)=p q$ and $\ell k\left(t^{\prime}, L_{g}^{\prime \prime} /\langle\bar{g}\rangle\right)=p q$, $\ell k\left(t^{\prime}, L_{g}^{\prime} /\langle\bar{g}\rangle\right)+\ell k\left(t^{\prime}, L_{g}^{\prime \prime} /\langle\bar{g}\rangle\right)=2 p q$. Hence by Lemma 5.4 and Claim 5.6, $L_{g}^{\prime}$ is a regular fiber. Similarly $L_{g}^{\prime \prime}$ is a regular fiber.

This completes the proof of Proposition 5.1(1).

(2) If two exceptional fibers in $(K ; r)$ cover the same exceptional fiber in $S^{3}$, then they have the same index. Since $(K ; r)$ contains three exceptional fibers whose indices are distinct from each other and for any Seifert fibration of $S^{3}$ there are at most two exceptional fibers, there exists an exceptional fiber $\tilde{t}$ in $(K ; r)$ which covers a regular fiber $t$. Then by Lemma 5.4 the index of $\tilde{t}$ is two and $t$ is a component of $\operatorname{Fix}(\bar{g}) /\langle\bar{g}\rangle$, and hence $\tilde{t}$ must be a component of $\operatorname{Fix}(\bar{g})$. This establishes Proposition 5.1 (2) $\quad \square$ (Proposition 5.1)

\section{Non-cyclic actions before/after Dehn surgeries.}

Throughout this section, we assume that $K$ is a hyperbolic knot in $S^{3}$, but not assume that $(K ; r)$ is Seifert fibered.

\subsection{Knots with Type I-C symmetry.}

Assume that $K$ has Type I-C symmetry. Hence we have a periodic diffeomorphism $f$ realizing the cyclic period 2 and a strong inversion $g$ such that they generate a finite subgroup $G=\langle f, g\rangle \subset \operatorname{Diff}^{*}\left(S^{3}, K\right)$. Since $h=g \circ f$ leaves $K$ invariant but reverses an orientation of $K$ and $h$ is periodic (because $\langle f, g\rangle$ is finite), $h^{2}$ fixes $K$ pointwisely. Thus by the Smith conjecture [39], $h^{2}$ is the identity map and so $h$ is also a strong inversion of $K$. Hence $g \circ f=(g \circ f)^{-1}=f^{-1} \circ g^{-1}=f \circ g$ and $G=\langle f, g\rangle$ is a dihedral group $\mathrm{D}_{2} \cong \mathbb{Z}_{2} \oplus \mathbb{Z}_{2}$ of order 4 . From the commutativity of $f$ and $g$, we have $f(\operatorname{Fix}(g))=\operatorname{Fix}(g)$ and $g(\operatorname{Fix}(f))=\operatorname{Fix}(f)$. In the following, we write $L_{f}=\operatorname{Fix}(\mathrm{f}), L_{g}=\operatorname{Fix}(\mathrm{g})$ and $L_{h}=\operatorname{Fix}(\mathrm{h})$, respectively.

Let us then consider the group action on the surgered manifold $(K ; r)$ induced from $G$ action on $\left(S^{3}, K\right)$. Let $N(K)$ be a $G$-invariant tubular neighborhood of $K$ in $S^{3}$. The restrictions $\left.f\right|_{E(K)}$ and $\left.g\right|_{E(K)}$ define a $\mathrm{D}_{2^{-}}$ action on $E(K)$. Then we can extend $\left.f\right|_{E(K)}$ and $\left.g\right|_{E(K)}$ over $(K ; r)$ so that 
each extension $\bar{f}, \bar{g}:(K ; r) \rightarrow(K ; r)$ has period 2 and they generate a subgroup $\bar{G} \subset \operatorname{Diff}((K ; r))$ isomorphic to $G \cong \mathrm{D}_{2}$. Since $L_{f}$ is contained in $E(K), L_{f}$ can be regarded as a circle in $(K ; r)$. To avoid a confusion, denote the image of $L_{f}$ in $(K ; r)$ by $L_{f}^{\prime}$. Recall from the beginning of Section 5 that the fixed point set $\operatorname{Fix}(\bar{g})$ consists of a single circle $L_{g}^{\prime}$ or two disjoint circles $L_{g}^{\prime} \cup L_{g}^{\prime \prime}$ in $(K ; r)$. Similarly $\operatorname{Fix}(\bar{h})$ consists of a single circle $L_{h}^{\prime}$ or two circles $L_{h}^{\prime} \cup L_{h}^{\prime \prime}$.

Then we have:

Lemma 6.1. The following (1), (2) and (3) are equivalent.

(1) $\operatorname{Fix}(\bar{f})=L_{f}^{\prime} \cup K^{*}\left(\operatorname{resp} . \operatorname{Fix}(\bar{f})=L_{f}^{\prime}\right)$.

(2) $\operatorname{Fix}(\bar{g})=L_{g}^{\prime} \cup L_{g}^{\prime \prime}\left(\operatorname{resp} . \operatorname{Fix}(\bar{g})=L_{g}^{\prime}\right)$.

(3) $\operatorname{Fix}(\bar{h})=L_{h}^{\prime} \cup L_{h}^{\prime \prime}\left(\operatorname{resp} . \operatorname{Fix}(\bar{h})=L_{h}^{\prime}\right)$.

Proof. We begin by showing:

Claim 6.2. $L_{f} \cap L_{g}=L_{f} \cap L_{h}$ and they consist of two points $P$ and $Q$.

Proof. First we show that $L_{f} \cap L_{g}$ consists of two points $P, Q$. Suppose that $L_{f} \cap L_{g}=\emptyset$. Since $g\left(L_{f}\right)=L_{f}, g$ defines a cyclic period of the trivial knot $L_{f}$, and hence $L_{f} \cup L_{g}$ is a Hopf link in $S^{3}$. Let $V_{f}$ be an $\langle h\rangle$-invariant tubular neighborhood of $L_{f}$; then $V_{g}=S^{3}-\operatorname{int} V_{f}$ is an $\langle h\rangle$-invariant tubular neighborhood of $L_{g}$. Referring the classification of semi-faithful involutions of a solid torus [26], $h$ has no fixed points on $V_{f}$ and $V_{g}$, thus $h$ is a free involution. This is a contradiction. Hence $L_{f} \cap L_{g} \neq \emptyset$. It follows that the periodic map $\left.g\right|_{L_{f}}: L_{f} \rightarrow L_{f}$ reverses the orientation of $L_{f}$, otherwise $\left.g\right|_{L_{f}}$ is the identity map. Thus $\left.g\right|_{L_{f}}$ fixes exactly two points in $L_{f}$, i.e., $L_{f} \cap L_{g}$ consists of two points $P, Q$. (Note that $L_{f}$ and $L_{g}$ are not tangent at both $P$ and $Q$. Because that $f$ is the identity map on $L_{f}$ and an reflection on $L_{g}$.)

Similarly $L_{f} \cap L_{h}$ consists of two points. Since $h=g \circ f$ fixes $P$ and $Q$, $L_{h}$ contains $P$ and $Q$. This implies that $L_{f} \cap L_{h}=\{P, Q\} . \quad \square($ Claim 6.2)

Recall that $L_{g} \cap E(K)$ consists of two arcs $c_{1}$ and $c_{2}$. If $c_{1} \ni P, Q$, then $\left.f\right|_{c_{1}}$ is the identity, contradicting the fact $L_{f} \cap L_{g}=\{P, Q\}$ (Claim 6.2). Hence, without loss of generality, we assume $c_{1} \ni P$ and $c_{2} \ni Q$. This then implies that if $\operatorname{Fix}(\bar{g})=L_{g}^{\prime} \cup L_{g}^{\prime \prime}$, then we may assume that $L_{g}^{\prime} \ni P$ and $L_{g}^{\prime \prime} \ni Q$. Similarly if $\operatorname{Fix}(\bar{h})=L_{h}^{\prime} \cup L_{h}^{\prime \prime}$, we assume that $L_{h}^{\prime} \ni P$ and $L_{h}^{\prime \prime} \ni Q$.

Claim 6.3. $\bar{f}$ leaves each component of $\operatorname{Fix}(\bar{g})$ and $\operatorname{Fix}(\bar{h})$ invariant. 
Proof. The commutativity $\bar{f} \circ \bar{g}=\bar{g} \circ \bar{f}$ implies $\bar{f}(\operatorname{Fix}(\bar{g}))=\operatorname{Fix}(\bar{g})$. If $\operatorname{Fix}(\bar{g})$ consists of two components $L_{g}^{\prime}, L_{g}^{\prime \prime}$, then since $L_{g}^{\prime} \ni P, L_{g}^{\prime \prime} \ni Q$, and $P, Q$ are fixed by $\bar{f}, \bar{f}\left(L_{g}^{\prime}\right)=L_{g}^{\prime}$ and $\bar{f}\left(L_{g}^{\prime \prime}\right)=L_{g}^{\prime \prime}$, as desired. The same argument shows that $\bar{f}$ leaves each component of $\operatorname{Fix}(\bar{h})$ invariant. $\quad \square$ (Claim 6.3)

Let us assume that $\operatorname{Fix}(\bar{f})=L_{f}^{\prime} \cup K^{*}$. If $\operatorname{Fix}(\bar{g})$ consists of a single component $L_{g}^{\prime}$, then from Lemma 6.3 , we can consider an involution $\left.\bar{f}\right|_{L_{g}^{\prime}}$ : $L_{g}^{\prime} \rightarrow L_{g}^{\prime}$. Since $L_{g}^{\prime}$ intersects $L_{f}^{\prime}$ in two points $P, Q$ and $L_{g}^{\prime}$ intersects $K^{*}$ in two points, $L_{g}^{\prime}$ intersect $\operatorname{Fix}(\bar{f})=L_{f}^{\prime} \cup K^{*}$ in four points. Hence $\left.\bar{f}\right|_{L_{g}^{\prime}}$ fixes at least four points and so it must be the identity map. Hence $L_{g}^{\prime} \subset \operatorname{Fix}(\bar{f})$, a contradiction. Thus $\operatorname{Fix}(\bar{g})=L_{g}^{\prime} \cup L_{g}^{\prime \prime}$. In the same manner we can show that $\operatorname{Fix}(\bar{h})=L_{h}^{\prime} \cup L_{h}^{\prime \prime}$.

Next assume that $\operatorname{Fix}(\bar{f})=L_{f}^{\prime}$, i.e., $\bar{f}$ fixes $L_{f}^{\prime}$ pointwisely but $\bar{f}$ is a free involution on $K^{*}$. Suppose for a contradiction that $\operatorname{Fix}(\bar{g})$ consists of two components $L_{g}^{\prime}$ and $L_{g}^{\prime \prime}$. Then $L_{g}^{\prime}$ intersects $L_{f}^{\prime}$ at $P$ and intersects $K^{*}$ in exactly one point. We recall that $\bar{f}\left(L_{g}^{\prime}\right)=L_{g}^{\prime}$ (Lemma 6.3). Thus $\bar{f}\left(L_{g}^{\prime} \cap K^{*}\right)=L_{g}^{\prime} \cap K^{*}$, and hence $\bar{f}$ has a fixed point on $K^{*}$, contradicting the fact that $\bar{f}$ is free on $K^{*}$. Thus $\operatorname{Fix}(\bar{g})=L_{g}^{\prime}$. By the same argument we can show that $\operatorname{Fix}(\bar{h})=L_{h}^{\prime}$. This completes the proof of Lemma 6.1.

$\square$ (Lemma 6.1)

\subsection{Knots with Type I-F symmetry.}

Let $K$ be a knot with Type I-F symmetry. Then we have a periodic diffeomorphism $f$ realizing the free period 2 and a strong inversion $g$ such that $G=\langle f, g\rangle$ is a finite subgroup of $\operatorname{Diff}^{*}\left(S^{3}, K\right)$. As in the previous subsection, $h=g \circ f$ is also a strong inversion of $K$ and $G=\langle f, g\rangle$ is a dihedral group $\mathrm{D}_{2} \cong \mathbb{Z}_{2} \oplus \mathbb{Z}_{2}$ of order 4 . Choosing a $G$-invariant tubular neighborhood $N(K)$ of $K$ in $S^{3}$, restrictions $\left.f\right|_{E(K)}$ and $\left.g\right|_{E(K)}$ define a finite group action on $E(K)$. Then we can extend $\left.f\right|_{E(K)}$ and $\left.g\right|_{E(K)}$ over $(K ; r)$ so that extensions $\bar{f}$ and $\bar{g}:(K ; r) \rightarrow(K ; r)$ generate a subgroup $\bar{G} \subset \operatorname{Diff}((K ; r))$ which is isomorphic to $G$. Since $f$ has period $2, \operatorname{Fix}(\bar{f})=K^{*}$ or $\operatorname{Fix}(\bar{f})=\emptyset$. Recall that $\operatorname{Fix}(\bar{g})=L_{g}^{\prime}$ or $L_{g}^{\prime} \cup L_{g}^{\prime \prime}$, and that $\operatorname{Fix}(\bar{h})=L_{h}^{\prime}$ or $L_{h}^{\prime} \cup L_{h}^{\prime \prime}$.

\section{Reflections on base orbifolds.}

Let $K$ be a knot with Type I-C or I-F symmetry. Then we have two involutions $f$ and $g ; f$ gives the cyclic or free period 2 and $g$ is a strong inversion. We use notation, $G, h, \bar{f}, \bar{g}, \bar{G}$, established in Subsections 6.1 and 6.2. Assume further that $\bar{G}$ preserves a Seifert fibration of $(K ; r)$. Then 
each element $\bar{\varphi} \in \bar{G}$ induces an orbifold isomorphism $\hat{\varphi}$ on the base orbifold $B$. Note the following relations: $\hat{h}=\hat{g} \circ \hat{f}, \hat{f}^{2}=\hat{g}^{2}=\hat{h}^{2}=$ id., which follow from the relations $\bar{h}=\bar{g} \circ \bar{f}, \bar{f}^{2}=\bar{g}^{2}=\bar{h}^{2}=$ id.. Hence we have also $\hat{f} \circ \hat{g}=\hat{g} \circ \hat{f}$. (In fact, $\hat{g} \circ \hat{f}=\hat{h}=\hat{h}^{-1}=\hat{f}^{-1} \circ \hat{g}^{-1}=\hat{f} \circ \hat{g}$.) Putting $\left\{\varphi_{1}, \varphi_{2}, \varphi_{3}\right\}=\{f, g, h\}$, we have: $\hat{\varphi}_{k}=\hat{\varphi}_{i} \circ \hat{\varphi}_{j}$ and $\hat{\varphi}_{i}{ }^{2}=\hat{\varphi}_{j}{ }^{2}=\hat{\varphi}_{k}{ }^{2}=$ id. for $\{i, j, k\}=\{1,2,3\}$. It should be noted here that the induced action of $\bar{G}$ on the base orbifold is not necessarily effective.

If $\hat{\varphi}_{i}$ is a reflection along a circle $C_{\varphi_{i}}(i=1,2)$, then the relation $\hat{\varphi}_{3}=$ $\hat{\varphi}_{1} \circ \hat{\varphi_{2}}$ implies that $\hat{\varphi}_{3}$ preserves an orientation of $|B|=S^{2}$.

Lemma 7.1. Suppose that both $\hat{\varphi}_{1}$ and $\hat{\varphi}_{2}$ are reflections on $|B|=S^{2}$ and that $\hat{\varphi}_{3} \neq \mathrm{id}$.. Then $C_{\varphi_{1}} \cap C_{\varphi_{2}}=\operatorname{Fix}\left(\hat{\varphi}_{3}\right)$, which consists of two points (Figure 1).

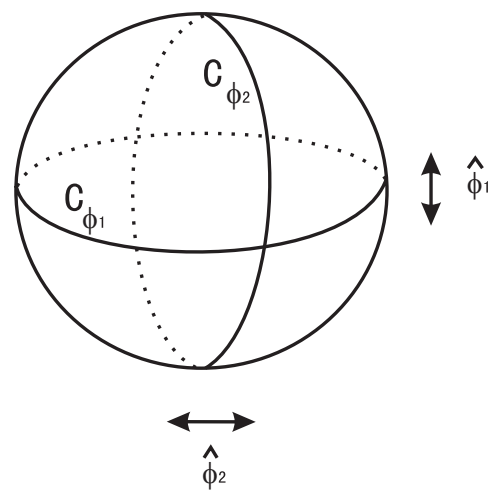

Figure 1

Proof. Since $\hat{\varphi_{3}}=\hat{\varphi_{2}} \circ \hat{\varphi}_{1}$, for any point $x \in C_{\varphi_{1}} \cap C_{\varphi_{2}}$, we have $\hat{\varphi}_{3}(x)=$ $\hat{\varphi}_{2}\left(\hat{\varphi}_{1}(x)\right)=\hat{\varphi}_{2}(x)=x$. Hence $C_{\varphi_{1}} \cap C_{\varphi_{2}}$ is contained in $\operatorname{Fix}\left(\hat{\varphi}_{3}\right)$. Since $\hat{\varphi}_{1}$ and $\hat{\varphi}_{2}$ are reflections, $\hat{\varphi}_{3}=\hat{\varphi}_{2} \circ \hat{\varphi}_{1}$ preserves an orientation of $|B|=S^{2}$. By the assumption $\hat{\varphi}_{3}$ is a nontrivial rotation and thus $\operatorname{Fix}\left(\hat{\varphi}_{3}\right)$ consists of two points. Hence $C_{\varphi_{1}} \cap C_{\varphi_{2}}$ consists of at most two points. On the other hand, if $C_{\varphi_{1}} \cap C_{\varphi_{2}}$ consists of at most one point, then $C_{\varphi_{2}}$ is lying in one side of $C_{\varphi_{1}}$. However, since $\hat{\varphi}_{1} \circ \hat{\varphi}_{2}=\hat{\varphi}_{2} \circ \hat{\varphi}_{1}, \hat{\varphi}_{1}\left(C_{\varphi_{2}}\right)=C_{\varphi_{2}}$ and hence $C_{\varphi_{2}}$ cannot lie in one side of $C_{\varphi_{1}}$. It follows that $C_{\varphi_{1}} \cap C_{\varphi_{2}}$ consists of two points and $C_{\varphi_{1}} \cap C_{\varphi_{2}}=\operatorname{Fix}\left(\hat{\varphi}_{3}\right)$. 
Lemma 7.2. Let $\varphi_{1}$ be a strong inversion of $K$. If $\hat{\varphi}_{1}$ is a reflection on $|B|=S^{2}$, then every cone point (i.e., $\pi\left(\right.$ exceptional fiber)) is lying on $C_{\varphi_{1}}$.

Proof. Recall first that $(K ; r) /\left\langle\bar{\varphi}_{1}\right\rangle \cong S^{3}$. Let us write $S^{2}-C_{\varphi_{1}}=D_{N} \cup D_{S}$, where $D_{N}, D_{S}$ are open disks and $D_{S}=\hat{\varphi}_{1}\left(D_{N}\right)$. If $D_{N}$ contains one cone point, then $(K ; r) /\left\langle\bar{\varphi}_{1}\right\rangle$ is a lens space other than $S^{3}$. If $D_{N}$ contains more than one cone point, then $(K ; r) /\left\langle\bar{\varphi}_{1}\right\rangle$ is a connected sum of lens spaces (for details, see [36]). Therefore $S^{2}-C_{\varphi_{1}}$ contains no cone points. This means that every cone point is lying on $C_{\varphi_{1}}$.

$\square$ (Lemma 7.2)

\section{Determining knot types using finite group actions.}

Throughout this section $K, G$ and $\bar{G}$ are as in Section 7. In what follows we will consider the situation that $(K ; r)$ is a Seifert fiber space over $S^{2}$ with more than three exceptional fibers whose Seifert fibration is preserved by $\bar{G}$.

The goal in this section is proving Lemma 8.5 in which we show under the above situation that if we have Condition $(\hat{f})$ or Condition $\left(K^{*}\right)$ defined below, then $K$ must be a composite knot $T_{p, q} \sharp T_{p, q}$.

\subsection{Locating exceptional fibers and dual knots.}

Let us consider the following condition.

Condition $(\hat{f}) \quad \hat{f}$ is a reflection along a circle $C_{f}$ on the base surface $|B|=$ $S^{2}$.

If we have Condition $(\hat{f})$, then the relation $\hat{h}=\hat{g} \circ \hat{f}$ implies that exactly one of $\hat{g}$ and $\hat{h}$ preserves an orientation and the other reverses an orientation of $|B|=S^{2}$. Without loss of generality, by exchanging roles of $g$ and $h$, we assume that $\hat{g}$ preserves an orientation (Condition $(\hat{g})$ ) and $\hat{h}$ reverses an orientation. Since $\operatorname{Fix}(\bar{h}) \neq \emptyset$, we have $\operatorname{Fix}(\hat{h}) \neq \emptyset$, so $\hat{h}$ is a reflection. Thus we have established:

Lemma 8.1. If we have Condition $(\hat{f})$, then we can assume that $\hat{g}$ preserves an orientation of $|B|=S^{2}$ (Condition $(\hat{g})$ ) and $\hat{h}$ is a reflection along a circle $C_{h}$.

The next lemma describes the configuration of cone points.

Lemma 8.2. Suppose that Condition $(\hat{f})$ holds. Then $C_{f}$ and $C_{h}$ intersect in exactly two points and cone points are lying on $C_{h}-C_{f}$, i.e., the configuration of cone points on $|B|=S^{2}$ is given by Figure 2 . 


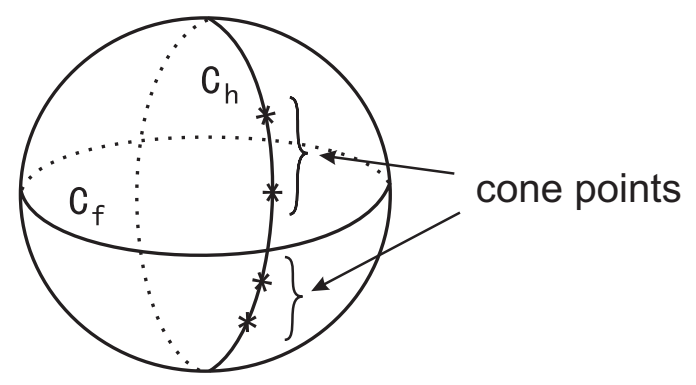

Figure 2

Proof. By the assumption and Lemma 8.1, we have also Condition $(\hat{g})$. Thus Proposition 5.1 (1) shows that $\operatorname{Fix}(\bar{g})$ consists of two components $L_{g}^{\prime}$ and $L_{g}^{\prime \prime}$ and each of which is a regular fiber in $(K ; r)$. Then it follows from Lemma 3.3 that $\hat{g}$ is not the identity map, and hence $\hat{g}$ is a nontrivial rotation. Therefore from Lemma 7.1 we see that $C_{f} \cap C_{h}=\operatorname{Fix}(\hat{g})$, which consists of two points $\pi\left(L_{g}^{\prime}\right)$ and $\pi\left(L_{g}^{\prime \prime}\right)$. Since $L_{g}^{\prime}$ and $L_{g}^{\prime \prime}$ are regular fibers, $\pi\left(L_{g}^{\prime}\right)$ and $\pi\left(L_{g}^{\prime \prime}\right)$ are not cone points. On the other hand, applying Lemma 7.2 to the strong inversion $h$, we can conclude that any cone point is lying on $C_{h}$. Hence all cone points are lying on $C_{h}-C_{f}$ as in Figure 2. $\square($ Lemma 8.2)

Now let us introduce Condition $\left(K^{*}\right)$ below.

Condition $\left(K^{*}\right)$ The dual knot $K^{*}$ is fixed pointwisely by $\bar{f}$.

Note that since $C_{f}$ contains no cone points (Lemma 8.2), $\pi^{-1}\left(C_{f}\right)$ is a torus.

Lemma 8.3. Suppose that Conditions $(\hat{f})$ and $\left(K^{*}\right)$ hold. Then the dual knot $K^{*}$ is contained in the vertical torus $\pi^{-1}\left(C_{f}\right)$, and $K^{*}$ intersects each Seifert fiber in $\pi^{-1}\left(C_{f}\right)$ without tangency.

Proof. We show first that $C_{f}=\pi(\operatorname{Fix}(\bar{f}))$. It is clear that $\pi(\operatorname{Fix}(\bar{f})) \subset C_{f}$. To show the converse, let us take a point $x \in C_{f}$. Then the fiber $t=\pi^{-1}(x)$ is invariant under $\langle\bar{f}\rangle$. If $\left.\bar{f}\right|_{t}: t \rightarrow t$ preserves an orientation of $t$, then $\hat{f}$ is orientation preserving. This contradicts Condition $(\hat{f})$. Hence $\left.\bar{f}\right|_{t}$ is orientation reversing and thus $\bar{f}$ fixes exactly two points in $t$. This implies that $t \cap \operatorname{Fix}(\bar{f}) \neq \emptyset$ and $x \in \pi(\operatorname{Fix}(\bar{f}))$. 
By Condition $\left(K^{*}\right)$ the dual knot $K^{*}$ is contained in $\operatorname{Fix}(\bar{f})$, thus $\pi\left(K^{*}\right) \subset C_{f}$. This means that $K^{*}$ is lying in the vertical torus $\pi^{-1}\left(C_{f}\right)$. To observe the last condition, assume for a contradiction that there is a fiber $t \subset \pi^{-1}\left(C_{f}\right)$ which is tangent to $K^{*}$. Then $\left.\bar{f}\right|_{t}: t \rightarrow t$ is an orientation preserving involution with nonempty fixed point set, and hence $\left.\bar{f}\right|_{t}=$ id.. Thus $t \subset \operatorname{Fix}(\bar{f})$, and this would then imply that $\hat{f}$ preserves an orientation of $|B|=S^{2}$, contradicting Condition $(\hat{f})$. Hence $K^{*}$ intersects each Seifert fiber in $\pi^{-1}\left(C_{f}\right)$ transversely in the vertical torus.

$\square$ (Lemma 8.3)

From Lemmas 8.2 and 8.3 we can deduce:

Lemma 8.4. Assume that $(K ; r)$ is a Seifert fiber space over $S^{2}$ with more than three exceptional fibers. If we have Conditions $(\hat{f})$ and $\left(K^{*}\right)$, then $K=T_{p, q} \sharp T_{p, q}$ for some torus knot $T_{p, q}$.

Proof. From Lemma 8.2, we can choose an $\langle\hat{f}\rangle$-invariant tubular neighborhood $A$ of $C_{f}$ in $S^{2}$ so that $A$ contains no cone points. Then $S^{2}-\operatorname{int} A$ consists of two disks $D_{N}$ and $D_{S}=\hat{f}\left(D_{N}\right)$, each of which contains at least two cone points. Since $C_{f}$ contains $\pi\left(K^{*}\right), \pi^{-1}(A)$ contains $N\left(K^{*}\right)$ for some tubular neighborhood $N\left(K^{*}\right)$. Since $A$ is an annulus without cone points, $\pi^{-1}(A)=$ $S^{1} \times S^{1} \times[-1,1]$. We may assume that $\pi^{-1}\left(C_{f}\right)=S^{1} \times S^{1} \times\{0\}$. The last assertion of Lemma 8.3 implies that $K^{*}$ is an essential loop in $\pi^{-1}\left(C_{f}\right)$, so there is a diffeomorphism of $\pi^{-1}(A)$ sending $K^{*}$ to $S^{1} \times\{x\} \times\{0\}$ for some $x \in S^{1}$. This implies that $\pi^{-1}(A)-\operatorname{int} N\left(K^{*}\right)$ is a 2 -fold composing space (i.e., $S^{1} \times$ [disk with 2-holes]). Consider a decomposition $S^{3}-\operatorname{int} N(K)=$ $(K ; r)-\operatorname{int} N\left(K^{*}\right)=\left(\pi^{-1}(A)-\operatorname{int} N\left(K^{*}\right)\right) \cup \pi^{-1}\left(D_{N}\right) \cup \pi^{-1}\left(D_{S}\right)$. Since $\pi^{-1}\left(D_{N}\right)\left(\cong \pi^{-1}\left(D_{S}\right)\right)$ is a Seifert fiber space in the knot exterior, we see that $\pi^{-1}\left(D_{N}\right)\left(\cong \pi^{-1}\left(D_{S}\right)\right)$ is a $(p, q)$-torus knot space ([28, VI.3.4 Lemma]) for some relatively prime integers $p$ and $q$. It follows that $K$ is a composite knot $T_{p, q} \sharp T_{p, q}$.

$\square$ (Lemma 8.4)

Remark. As shown in [45], in general we cannot choose a periodic diffeomorphism $f$ realizing cyclic (resp. free) period and a strong inversion $g$ so that they generate a finite subgroup of $\operatorname{Diff}^{*}\left(S^{3}, K\right)$. For a composite knot $K=T_{p, q} \sharp T_{p, q}$, we can choose such diffeomorphisms $f$ and $g$ so that they generate a finite subgroup of $\operatorname{Diff}^{*}\left(S^{3}, K\right)$.

\subsection{Equivalence of Condition $(\hat{f})$ and Condition $\left(K^{*}\right)$.}

In this subsection we prove that Condition $(\hat{f})$ and Condition $\left(K^{*}\right)$ are actually equivalent under the assumption that $(K ; r)$ is a Seifert fiber space 
over $S^{2}$ with more than three exceptional fibers, see Lemma 8.6 below. Hence Lemma 8.4 can be improved in the following form.

Lemma 8.5. Assume that $(K ; r)$ is a Seifert fiber space over $S^{2}$ with more than three exceptional fibers. If we have Condition $(\hat{f})$ or Condition $\left(K^{*}\right)$, then $K=T_{p, q} \sharp T_{p, q}$.

Lemma 8.6. Assume that $(K ; r)$ is a Seifert fiber space over $S^{2}$ with more than three exceptional fibers. Then Condition $(\hat{f})$ implies Condition $\left(K^{*}\right)$, and vice versa.

Proof. Note that since $(K ; r)$ is a Seifert fiber space over $S^{2}$ with more than three exceptional fibers, $K$ is not a torus knot.

First we suppose that $f$ gives a cyclic period 2 of $K$. Let us assume that Condition $(\hat{f})$ holds. Then from Lemma 8.1, we have also Condition $(\hat{g})$. Since $(K ; r)$ contains more than three exceptional fibers, by Proposition 5.1, $\operatorname{Fix}(\bar{g})$ consists of two components. Then Lemma 6.1 shows that $K^{*}$ is a component of $\operatorname{Fix}(\bar{f})$. Hence we have Condition $\left(K^{*}\right)$. Conversely suppose that Condition $\left(K^{*}\right)$ holds. Since $K$ is not a torus knot, the argument in the proof of Lemma 4.1 shows that $\hat{f}$ reverses an orientation of $S^{2}$. Since $\operatorname{Fix}(\bar{f}) \neq \emptyset, \hat{f}$ has also nonempty fixed point set and thus $\hat{f}$ is a reflection, i.e., Condition $(\hat{f})$ holds.

Next we suppose that $f$ gives a free period 2 of $K$. If we have Condition $(\hat{f})$, then from the argument in the proof of Lemma 8.3 we see that $\operatorname{Fix}(\bar{f}) \neq \emptyset$. Hence $\operatorname{Fix}(\bar{f})=K^{*}$ (cf. the first paragraph of Subsection 4.1), and we have Condition $\left(K^{*}\right)$. Conversely let us assume Condition $\left(K^{*}\right)$. Recalling from Lemma 4.1, we see that $\hat{f}$ reverses an orientation of $S^{2}$. Since $\operatorname{Fix}(\bar{f}) \neq \emptyset, \operatorname{Fix}(\hat{f}) \neq \emptyset$, and hence $\hat{f}$ is a reflection, i.e., Condition $(\hat{f})$ holds.

$\square($ Lemma 8.4)

\section{Toroidal Seifert fibered surgeries for hyperbolic knots.}

We are now in a position to prove Theorem 1.1 for hyperbolic knots with Type I-C symmetry (Theorem 9.1) and hyperbolic knots with Type I-F symmetry (Theorem 9.2).

\subsection{Surgeries on knots with Type I-C symmetry.}

Theorem 9.1. Let $K$ be a hyperbolic knot with Type I-C symmetry. Then $(K ; r)$ cannot be a toroidal Seifert fiber space. 
Proof. Since $K$ has Type I-C symmetry, we have a finite subgroup $G$ of $\operatorname{Diff}^{*}\left(S^{3}, K\right)$ generated by an involution $f$ realizing the cyclic period 2 and a strong inversion $g$.

Assume for a contradiction that $(K ; r)$ is a toroidal Seifert fiber space. Then the base surface of $(K ; r)$ is the 2 -sphere ([36, Theorem 1.3$])$ and $(K ; r)$ contains more than three exceptional fibers (Lemma 4.11).

As in Subsection 6.1 the action of $G$ on $S^{3}$ induces an action of $\bar{G}$ on $(K ; r)$, where $\bar{G}$ is generated by $\bar{f}, \bar{g}$.

From Lemma 3.1 (1) we choose a Seifert fibration of $(K ; r)$ which is invariant under $\bar{G}$.

If $L_{f}^{\prime}$, the image of the axis $L_{f}=\operatorname{Fix}(f)\left(\subset S^{3}\right)$, is a fiber in $(K ; r)$, then by [35, Proposition 5.1] $K$ is a torus knot or a cable of a torus knot. This contradicts the hyperbolicity of $K$. Hence $L_{f}^{\prime}$ is not a fiber in $(K ; r)$ and the argument in the proof of Claim 4.16 shows that $\hat{f}: S^{2} \rightarrow S^{2}$ is a reflection, i.e., Condition $(\hat{f})$ holds. Now it follows from Lemma 8.5 that $K=T_{p, q} \sharp T_{p, q}$. This contradicts the hyperbolicity of $K$ again and establishes Theorem 9.1.

$\square$ (Theorem 9.1)

\subsection{Surgeries on knots with Type I-F symmetry.}

Theorem 9.2. Let $K$ be a hyperbolic knot with Type I-F symmetry. Then $(K ; r)$ cannot be a toroidal Seifert fiber space.

Proof. As in the proof of Theorem 9.1, let $G$ be a finite subgroup of $\operatorname{Diff}^{*}\left(S^{3}, K\right)$ generated by an involution $f$ realizing the free period 2 and a strong inversion $g$.

Assume for a contradiction that $(K ; r)$ is a toroidal Seifert fiber space. Then by Theorem 4.5 the base surface of $(K ; r)$ is the 2 -sphere and $(K ; r)$ contains more than three exceptional fibers (Lemma 4.11).

Let $\bar{G}$ be a finite group generated by $\bar{f}$ and $\bar{g}$ (see Subsection 6.2 ). We choose a Seifert fibration of $(K ; r)$ which is preserved by $\bar{G}$ (Lemma 3.1 (1)).

If we have Condition $(\hat{f})$ or Condition $\left(K^{*}\right)$, then by Lemma $8.5 K=$ $T_{p, q} \sharp T_{p, q}$. This contradicts the hyperbolicity of $K$. Hence $\operatorname{Fix}(\bar{f})=\emptyset$ and $\hat{f}$ is not a reflection.

Lemma 9.3. The fixed point set $\operatorname{Fix}(\hat{f})$ is not empty.

Proof. To the contrary suppose that $\operatorname{Fix}(\hat{f})=\emptyset$. Then $\hat{f}$ is orientation reversing. Since the period $p$ is $2, \hat{f}$ is an antipodal map and there exists an $\hat{f}$-essential loop $c$ so that $\hat{f}(c) \cap c=\emptyset$. This contradicts Lemma 4.3. 
Since $\hat{f}$ is not a reflection, by Lemma $9.3, \hat{f}$ is the identity map or a rotation of order 2 (see Section 3 ).

If $\hat{f}$ is the identity map, then we have an $\hat{f}$-essential loop $c$ on $B$. This contradicts Lemma 4.3 .

It follows that $\hat{f}$ is a rotation of order 2 with $\operatorname{Fix}(\hat{f})=\{P, Q\}$.

Since $\hat{h}=\hat{g} \circ \hat{f}$, there are two possibilities:

(1) Both $\hat{g}$ and $\hat{h}$ preserve an orientation of $|B|=S^{2}$.

(2) Both $\hat{g}$ and $\hat{h}$ reverse an orientation of $|B|=S^{2}$.

Suppose (1) occurs. Then Condition $(\hat{g})$ holds. It follows from Proposition $5.1(1)$ that $\operatorname{Fix}(\bar{g})$ consists of two regular fibers, and then by Lemma $3.3, \hat{g} \neq \mathrm{id}$.. Hence $\hat{g}$ is a rotation of order 2 such that Fix $(\hat{g})$ consists of two regular points. Exchanging the roles of $g$ and $h$, we see that $\hat{h}$ is also a rotation of order 2 such that $\operatorname{Fix}(\hat{h})$ consists of two regular points.

Lemma 9.4. There exists a cone point $z$ in $B-\operatorname{Fix}(\hat{f}) \cup \operatorname{Fix}(\hat{g}) \cup \operatorname{Fix}(\hat{h})$.

Proof. Recall that the base orbifold $B$ contains more than three cone points. Since $\operatorname{Fix}(\hat{g}) \cup \operatorname{Fix}(\hat{h})$ contains no cone points, $B-\operatorname{Fix}(\hat{g}) \cup \operatorname{Fix}(\hat{h})$ contains more than three cone points. Since $\operatorname{Fix}(\hat{f})$ consists of exactly two points, we can find a cone point $z$ in $B-\operatorname{Fix}(\hat{f}) \cup \operatorname{Fix}(\hat{g}) \cup \operatorname{Fix}(\hat{h}) . \quad \square($ Lemma 9.4)

Then we have the following, which contradicts Lemma 4.12.

Lemma 9.5. Let $z$ be a cone point given by Lemma 9.4. Then any two of $z, \hat{f}(z), \hat{g}(z)$ and $\hat{h}(z)$ are distinct. Thus there are four cone points of the same index.

Proof. Since $z \in B-\operatorname{Fix}(\hat{f}) \cup \operatorname{Fix}(\hat{g}) \cup \operatorname{Fix}(\hat{h}), \hat{f}(z) \neq z, \hat{g}(z) \neq z$ and $\hat{h}(z) \neq z$. If $\hat{f}(z)=\hat{g}(z)$, then $\hat{h}(z)=\hat{g} \circ \hat{f}(z)=z$, a contradiction. Similarly we can show other inequalities. Hence there are four cone points $z, \hat{f}(z)$, $\hat{g}(z), \hat{h}(z)$, which have the same index.

$\square$ (Lemma 9.5)

Finally suppose $(2)$ happens. Since $\operatorname{Fix}(\bar{g}) \neq \emptyset$ and $\operatorname{Fix}(\bar{h}) \neq \emptyset$, we have also $\operatorname{Fix}(\hat{g}) \neq \emptyset$ and $\operatorname{Fix}(\hat{h}) \neq \emptyset$. Thus both $\hat{g}$ and $\hat{h}$ are reflections. In addition, since $\hat{f}$ is a nontrivial rotation, from Lemma 7.1 we see that $C_{g} \cap C_{h}=\operatorname{Fix}(\hat{f})$, which consists of two points $P$ and $Q$. Applying Lemma 7.2 to strong inversions $g, h$ of $K$, we can conclude that all cone points are lying on $C_{g} \cap C_{h}$ (Figure 3 ).

It follows that the base orbifold $B$ contains at most two cone points $P$ and $Q$, a contradiction. The proof of Theorem 9.2 is now complete. $\square$ (Theorem $9.2)$ 


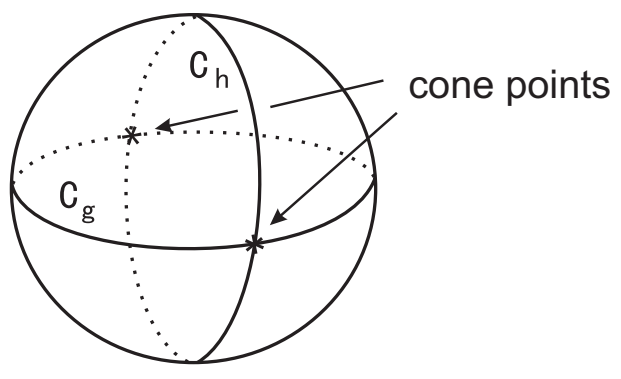

Figure 3

\section{Toroidal Seifert fibered surgeries for non-hyperbolic knots.}

The purpose in this section is proving:

Theorem 1.5. Let $K$ be a non-hyperbolic knot with symmetry which is not a strong inversion. If $(K ; r)$ is a toroidal Seifert fiber space, then $K$ is a trefoil knot $(r=0), T_{p, q} \sharp T_{p, q}(r=2 p q)$ or $T_{p, q} \sharp T_{-p, q}(r=0)$.

Proof. Following Thurston's uniformization theorem [51]([39]) and the torus theorem [28], [29] if a knot $K$ is non-hyperbolic, then $K$ is a torus knot or a satellite knot, i.e., a knot whose exterior contains an incompressible torus which is not boundary-parallel. For a satellite knot $K$, a solid torus in $S^{3}$ bounded by such a torus is called a companion solid torus.

We recall from [33, Theorem 1.2] that if $K$ is a non-hyperbolic knot and $(K ; r)$ is a toroidal Seifert fiber space, then one of the following occurs.

(i) $K$ is a trefoil knot $(r=0)$,

(ii) $K$ is the $(2 p q \pm 1,2)$-cable of a $(p, q)$-torus knot $(r=4 p q)$,

(iii) $K$ is a connected sum of two torus knots $T_{p, q} \sharp T_{p^{\prime}, q^{\prime}}\left(r=p q+p^{\prime} q^{\prime}\right)$, or

(iv) $K$ has a companion solid torus $V$ whose core is a torus knot and $V-K$ admits a complete hyperbolic structure in its interior $(r=$ an integer).

Claim 10.1. Among knots described above, only $T_{p, q} \sharp T_{-p, q}$ is amphicheiral.

Proof. Let $\varphi$ be an orientation reversing diffeomorphism of $\left(S^{3}, K\right)$. Since a tubular neighborhood $N(K)$ of $K$ is unique up to isotopy, we may assume $\varphi(N(K))=N(K)$. Then the uniqueness of the torus decomposition of 
$E(K)$, we can assume further that the family of decomposing tori is invariant under $\varphi$. Since a torus knot space cannot admit an orientation reversing selfdiffeomorphism, $\varphi$ cannot leave a torus knot space invariant. It follows that only $T_{p, q} \sharp T_{-p, q}(r=0)$ is an amphicheiral knot among knots of types (i)-(iv).

$\square($ Claim 10.1)

Henceforth we consider symmetries which preserve an orientation of $S^{3}$.

Since a satellite knot of type (ii) has a cyclic surgery ( $4 p q \pm 1$-surgery), the knot has no symmetry other than a strong inversion [53]. Assume that $K=T_{p, q} \sharp T_{p^{\prime}, q^{\prime}}$. If $K$ has a free period, then [3, Lemma 2.1 (3)] implies that $T_{p, q} \cong T_{p^{\prime}, q^{\prime}}$, i.e., $K=T_{p, q} \sharp T_{p, q}$. If $K$ has a cyclic period, then the period is 2 by Theorem 2.4 ([35, Theorem 1.5]), and the argument in the proof of [26, Theorem 4.1] shows that $T_{p, q} \cong T_{p^{\prime}, q^{\prime}}$, i.e., $K=T_{p, q} \sharp T_{p, q}$.

Thus it is sufficient to prove that satellite knots of type (iv) with a symmetry other than a strong inversion cannot admit a toroidal Seifert fibered surgery.

We begin by showing the following.

Proposition 10.2. Let $K$ be a satellite knot of winding number zero, i.e., $K$ has a companion solid torus $V$ in which $K$ is homologous to zero. Then $K$ admits no Seifert fibered surgeries.

Proof. Note that if $(K ; r)$ is a small Seifert fiber space, then $K$ has a companion solid torus $V$ whose core is a simple knot (i.e., a torus knot or a hyperbolic knot) in $S^{3}$, and $K(\subset V)$ is a 0 or 1-bridge braid in $V$ in the sense of Gabai [18]([34, Proposition 2.2]). Clearly $K$ has nonzero winding number in this case. Henceforth we consider the case where $(K ; r)$ is a toroidal Seifert fiber space. Except for (iv), $K$ has nonzero winding number. Let us assume that $K$ is in (iv) and assume for a contradiction that $K$ is homologous to zero in $V$. Then we have a Thurston norm minimizing (orientable) surface $S$ in $V-\operatorname{int} N(K)$ such that $\partial S$ is a meridian, which remains norm minimizing, hence incompressible in $V(K ; r)$ (see [16, Corollary 2.5]). Now recall from [33] that $V(K ; r)$ is a boundary-irreducible Seifert fiber space admitting a Seifert fibration which is the restriction of that of $(K ; r)$. This implies that a regular fiber on $\partial V(K ; r)(=\partial V)$ intersects a meridian of $V$ exactly once, and hence that $\partial S$ can be isotoped so that $\partial S$ intersects a regular fiber exactly once. Since $S$ is essential in the Seifert fiber space $V(K ; r), S$ is horizontal (i.e., transverse to fibers) or vertical (i.e., consists of fibers). However the latter is impossible, because $\partial S$ intersects a regular fiber exactly once. Thus restricting the Seifert fibration $\pi: V(K ; r) \rightarrow B\left(|B|=D^{2}\right.$ or Möbius band) to $S$, we obtain a branched covering $\left.\pi\right|_{S}: S \rightarrow B ;\left.\pi\right|_{\partial S}$ is one to one, 
because $\partial S$ intersects each fiber in $\partial V(K ; r)$ exactly once. Therefore the degree of the branched covering is one, i.e., $\left.\pi\right|_{S}$ is a diffeomorphism. This implies that $S$ is a disk, which would imply that $K$ is contained in a 3-ball in $V$, a contradiction.

$\square$ (Proposition 10.2)

Remark. A null-homologous knot in a solid torus may admit a Seifert fibered surgery. For instance, take a Whitehead link $k_{1} \cup k_{2} ; V=S^{3}-\operatorname{int} N\left(k_{2}\right)$ and $K=k_{1}$ is a knot in $V$ which is homologous to zero. Then $V(K ;-1)$ is a Seifert fiber space of type $D^{2}(2,3)$.

Let $K$ be a satellite knot of type (iv) and $f: S^{3} \rightarrow S^{3}$ a periodic diffeomorphism giving the cyclic or free period $p$ of $K$. If $f$ gives the cyclic period, then by Theorem 2.4 ([35, Theorem 1.5]), we may assume $p=2$. Let $N(K)$ be an $\langle f\rangle$-invariant tubular neighborhood of $K$. By the existence of invariant torus decomposition of $S^{3}-\operatorname{int} N(K)$ ([31]) and its uniqueness up to isotopy ([28], [29]) we can assume up to conjugation that $f(V)=V$. Thus we can obtain (after conjugation) a periodic diffeomorphism $\left.f\right|_{V}:(V, K) \rightarrow$ $(V, K)$ of period $p \geq 2$.

Claim 10.3. $\left.f\right|_{V}:(V, K) \rightarrow(V, K)$ is freely periodic, i.e., $\left.f\right|_{V}$ is a periodic diffeomorphism such that $\operatorname{Fix}\left(\left(\left.f\right|_{V}\right)^{i}\right)=\emptyset$ for $i=1, \ldots, p-1$.

Proof. Note that $\left.f\right|_{V}$ leaves $K$ invariant and preserves an orientation of $K$. If $f:\left(S^{3}, K\right) \rightarrow\left(S^{3}, K\right)$ is a freely periodic diffeomorphism, then $\left.f\right|_{V}$ is also freely periodic. Let us assume that $f:\left(S^{3}, K\right) \rightarrow\left(S^{3}, K\right)$ is a cyclically periodic diffeomorphism with fixed point set $C=\operatorname{Fix}(f)$ which is a trivial knot by the Smith conjecture [39]. As we mentioned above, we can assume further $p=2$. Suppose that $C \cap V \neq \emptyset$. Then referring [26], we can conclude that $C \cap V$ consists of two properly embedded arcs or $C$ is a core of $V$. If the former case happens, then $\left(\left.f\right|_{V}\right)_{*}: H_{1}(V) \rightarrow H_{1}(V)$ sends $z$ to $-z$ for a generator $z \in H_{1}(V)$. Since $K$ is not homologous to zero (Lemma 10.2), $f$ reverses an orientation of $K$, a contradiction. If the latter case happens, then $C$ is knotted in $S^{3}$, a contradiction.

$\square($ Claim 10.3)

Denote by $\overline{\left.f\right|_{V}}$ the periodic diffeomorphism of $V(K ; r)$ with period $p \geq 2$ induced from $\left.f\right|_{V}$.

Note that $V(K ; r)$ admits a Seifert fibration which is the restriction of that of $(K ; r)([33])$, and that the base surface is a disk or a Möbius band. If the base surface of $V(K ; r)$ is a Möbius band, then the base surface of $(K ; r)$ is $\mathbb{R} P^{2}$. Hence by [34, Theorem 1.3] $K$ is a $(2 p q \pm 1,2)$-cable of a $(p, q)$-torus knot. This contradicts that $K$ is of type (iv). Thus with respect to the restricted Seifert fibration, $V(K ; r)$ is Seifert fibered over the disk with at least two exceptional fibers. 
Lemma 10.4. There exists an $\left\langle\overline{\left.f\right|_{V}}\right\rangle$-invariant Seifert fibration of $V(K ; r)$.

Proof. If $V(K ; r)$ is not of type $D^{2}(2,2)$ (a twisted I-bundle over the Klein bottle), then the Seifert fibration of $V(K ; r)$ is unique up to isotopy [27], and hence by [31] there is an $\left\langle\overline{\left.f\right|_{V}}\right\rangle$-invariant Seifert fibration.

Now we show that $V(K ; r)$ cannot be a Seifert fiber space of type $D^{2}(2,2)$. To do so assume for a contradiction that $V(K ; r)$ is of such type. Then attach a solid torus $W$ to $V$ so that the meridian of $W$ is identified with $p q[$ meridian of $V]+[$ longitude of $V]$; recall that the core of $V$ is a $(p, q)$-torus knot. As a result we obtain $S^{3}$ and a new knot $K_{p q}^{\prime}$ in this new 3-sphere. By the construction $\left(K_{p q}^{\prime} ; \gamma\right) \cong \mathbb{R} P^{3} \sharp \mathbb{R} P^{3}$, it is impossible for homological reason.

$\square$ (Lemma 10.4)

Let us choose a Seifert fibration of $V(K ; r)$ preserved by $\left\langle\overline{\left.f\right|_{V}}\right\rangle$. By taking some power of $\left.f\right|_{V}$, we may assume that $\left.f\right|_{V}$ has a prime period $p \geq 2$, so that $\operatorname{Fix}\left(\overline{\left.f\right|_{V}}\right)=K^{*}$ or $\operatorname{Fix}\left(\left(\overline{\left.f\right|_{V}}\right)^{i}\right)=\emptyset$ for $1 \leq i \leq p-1$.

Case $1: \operatorname{Fix}\left(\overline{\left.f\right|_{V}}\right)=K^{*}$.

Since $\overline{\left.f\right|_{V}}$ preserves the Seifert fibration of $V(K ; r)$, we have the induced orbifold isomorphism $\widehat{\left.f\right|_{V}}: B \rightarrow B$, where $|B|$ is a disk.

If $\widehat{\left.f\right|_{V}}$ is orientation preserving, then the argument in the proof of Lemma 3.2 shows that $\operatorname{Fix}\left(\overline{\left.f\right|_{V}}\right)=K^{*}$ is a fiber. Hence $V-K=V(K ; r)-K^{*}$ is Seifert fibered, contradicting the hyperbolicity of $K$ in $V$. Suppose that $\widehat{\left.f\right|_{V}}$ reverses an orientation of $|B|=D^{2}$. Then $\widehat{\left.f\right|_{V}}$ is a reflection along a properly embedded arc $\operatorname{Fix}\left(\widehat{\left.f\right|_{V}}\right)=\ell$ in $D^{2}$. Let $x$ be a point in $\ell \cap \partial D^{2}$. Then $\overline{\left.f\right|_{V}}$ acts on the fiber $t=\pi^{-1}(x)$ as a reflection. Thus $\overline{\left.f\right|_{V}}$ has two fixed points on $t$. Since $t$ is embedded in $\partial V(K ; r), \operatorname{Fix}\left(\overline{\left.f\right|_{V}}\right) \cap \partial V(K ; r)$ is nonempty. This contradicts that $\operatorname{Fix}\left(\overline{\left.f\right|_{V}}\right)$, which is the dual knot $K^{*}$, is entirely contained in the interior of $V(K ; r)$.

Case $2: \operatorname{Fix}\left(\left(\overline{\left.f\right|_{V}}\right)^{i}\right)=\emptyset$ for $1 \leq i \leq p-1$.

Since $\operatorname{Fix}\left(\left(\left.f\right|_{V}\right)^{i}\right)=\emptyset$ and $\left.\operatorname{Fix}\left(\overline{\left(\left.f\right|_{V}\right.}\right)^{i}\right)=\emptyset$ for $1 \leq i<p$, we have covering projections $\rho: V \rightarrow V_{f}=V /\left\langle\left. f\right|_{V}\right\rangle$ and $\bar{\rho}: V(K ; \gamma) \rightarrow V(K ; \gamma) /\left\langle\overline{\left.f\right|_{V}}\right\rangle$. Let $K_{f}$ be the image $\rho(K)$ in the solid torus $V_{f}=V /\left\langle\left. f\right|_{V}\right\rangle$ and $\gamma$ the slope corresponding with $r$. Then the quotient manifold $V(K ; \gamma) /\left\langle\overline{\left.f\right|_{V}}\right\rangle$ can be regarded as $V_{f}\left(K_{f}, \gamma_{f}\right)$ for some surgery slope $\gamma_{f}$. Thus we have the diagram:

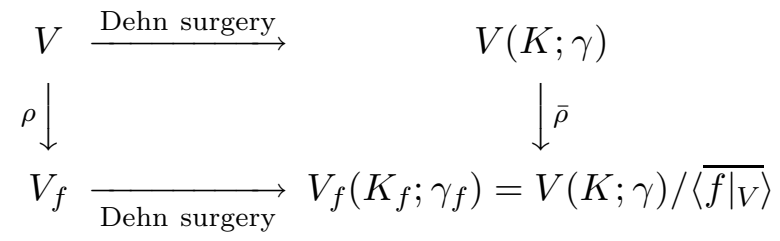


Since $V(K ; \gamma)$ is Seifert fibered and $\overline{\left.f\right|_{V}}$ preserves the Seifert fibration, $V(K ; \gamma) /\left\langle\overline{\left.\left.f\right|_{V}\right\rangle}\right.$ is also Seifert fibered. Then [35, Theorem 1.2] asserts that either (i) $K_{f} \subset V_{f}\left(\cong S^{1} \times D^{2}\right)$ is a core of $V_{f}$ or a cable of a 0-bridge braid in $V_{f}$, or (ii) $\gamma_{f}$ is integral, i.e., $\Delta\left(\gamma_{f}, \mu_{f}\right)$, the minimal geometric intersection number of $\gamma_{f}$ and the meridional slope $\mu_{f}$, is 1 . On the other hand, $\Delta\left(\gamma_{f}, \mu_{f}\right)=p(\geq 2)$, because $\operatorname{Fix}\left(\left(\overline{\left.f\right|_{V}}\right)^{i}\right)=\emptyset$ for $1 \leq i \leq p-1$; see the proof of Claim 4.4. Thus (ii) cannot happen. Since $K$ is hyperbolic in $V$ and $V-K \rightarrow V_{f}-K_{f}$ is covering, $V_{f}-K_{f}$ is irreducible, atoroidal and not Seifert fibered. This excludes the first possibility (i).

It follows that a satellite knot of type (iv) has no toroidal Seifert fibered surgery. Hence only a trefoil knot, $T_{p, q} \sharp T_{p, q}$ and $T_{p, q} \sharp T_{-p, q}$ are non-hyperbolic knots having a symmetry other than a strong inversion and admitting toroidal Seifert fibered surgeries, as claimed in the theorem.

$\square$ (Theorem 1.5)

Those knots described in the theorem are strongly invertible and they have further symmetries. In fact, a trefoil knot has also cyclic periods and free periods, and 0-surgery yields a toroidal Seifert fiber space of type $S^{2}(2,3,6)$; a composite knot $T_{p, q \sharp} T_{p, q}$ admits also a cyclic period and a free period and $\left(T_{p, q} \sharp T_{p, q} ; 2 p q\right)$ is a toroidal Seifert fiber space of type $S^{2}(|p|, q,|p|, q)$; a composite knot $T_{p, q} \sharp T_{-p, q}$ is invariant under an orientation reversing involution of $S^{3}$, and $\left(T_{p, q} \sharp T_{-p, q} ; 0\right)$ is a toroidal Seifert fiber space of type $S^{2}(|p|, q,|p|, q)$.

\section{Projective Seifert fibered surgeries on symmetric knots.}

In this section we complete the proof of Theorem 1.3.

Theorem 1.3. Let $K$ be a hyperbolic knot with symmetry which is not a strong inversion. Then there exists no surgery on $K$ yielding a Seifert fiber space over $\mathbb{R} P^{2}$.

Proof. Let $\varphi$ be a symmetry of $K$, i.e., a periodic diffeomorphism of $\left(S^{3}, K\right)$, which is not a strong inversion. First suppose that $\varphi$ preserves an orientation of $S^{3}$. Then it gives a cyclic period, a free period or a semi-free period (see Section 2). Recall that if $\varphi$ gives a semi-free period, then some power of $\varphi$ gives a cyclic period. Theorem 1.3 follows from [36, Theorem 1.3] if $K$ has a cyclic period and Theorem 4.5 if $K$ has a free period. Suppose that $\varphi$ reverses an orientation of $S^{3}$, i.e., $K$ is amphicheiral. Then there is an orientation reversing diffeomorphism $\bar{\varphi}:(K ; m) \rightarrow(K ;-m)$. Since by Lemma $4.6 m=4 k$ for some $k(\neq 0)$, thus the distance of two slopes $m$ and $(-m)$ is greater than or equal to 8 . 
Assume first that $(K ; m)$ is Seifert fibered over $\mathbb{R} P^{2}$ with at least two exceptional fibers so that $(K ; m)$ is toroidal. Since both $m$ and $(-m)$-surgeries are toroidal surgeries and the distance of their surgery slopes $|2 m|$ is greater than or equal to $8,\left[21\right.$, Theorem 1.1] implies that $S^{3}-\operatorname{int} N(K)$ is a figureeight knot exterior (and $m=4$ ), and hence, $K$ is the figure-eight knot. But since the figure-eight knot has a cyclic period 2 (resp. genus one), by [36, Theorem 1.3] (resp. [49]) it has no surgery producing a Seifert fiber space over $\mathbb{R} P^{2}$, a contradiction.

Next suppose that $(K ; m)$ is Seifert fibered over $\mathbb{R} P^{2}$ with at most one exceptional fiber. Then $(K ; m)$ is also a Seifert fiber space of type $S^{2}(2,2, x)$ $(x \geq 1)$, and hence, $\pi_{1}((K ; m))$ is a cyclic group or a D-type finite group in $[6]([44, \mathrm{p} .131])$. Since $(K ; m)$ is diffeomorphic to $(K ;-m)$, if $m$-surgery is a cyclic surgery (resp. a D-type finite surgery), then $(-m)$-surgery is also a cyclic surgery (resp. a D-type finite surgery) and the distance of their surgery slopes is greater than or equal to 8. However the cyclic surgery theorem [11] shows that the distance between two cyclic surgery slopes is less than or equal to 1 and [6, Corollary 1.6 (1)(ii)] shows that there is at most one D-type finite surgery. This contradiction completes the proof of Theorem 1.3.

$\square$ (Theorem 1.3)

\section{Small Seifert fibered surgeries.}

The purpose of this final section is proving Theorem 1.7.

Theorem 1.7. Let $K$ be a strongly invertible knot with cyclic period 2 .

(1) If $(K ; r)$ is a small Seifert fiber space of type $S^{2}\left(n_{1}, n_{2}, n_{3}\right)$ with $2<$ $n_{1}<n_{2}<n_{3}$, then $K$ is a torus knot or a cable of a torus knot.

(2) If $(K ; r)$ is a small Seifert fiber space of type $S^{2}\left(2, n_{2}, n_{3}\right)$ such that $\left(n_{2}, n_{3}\right) \neq 1$ and $\left(n_{2}, n_{3}\right) \neq(3,3)$, then $K$ is a torus knot or a cable of a torus knot.

Before proving the theorem, we give some examples of Seifert fiber spaces obtained by surgeries on strongly invertible knots with cyclic period 2 .

Examples. Let $K$ be the figure-eight knot. Then $(K ; r)$ is a Seifert fiber space of type $S^{2}(2,3,7)$ for $r= \pm 1$, type $S^{2}(2,4,5)$ for $r= \pm 2$. In these examples the indices of three exceptional fibers are distinct from each other; the smallest one is 2 (Theorem 1.7(1)) and the others are relatively prime (Theorem $1.7(2))$. \pm 3 -surgery on $K$ yields also a Seifert fiber space of type 
$S^{2}(3,3,4)$. In this example, every exceptional fiber has index $>2$, but two of them have the same index (Theorem 1.7(1)).

On the other hand, for a $(3,4)$-torus knot $T_{3,4},\left(T_{3,4} ; 7\right)$ is a Seifert fiber space of type $S^{2}(3,4,5)$ and for a $(2,3)$-torus knot $T_{2,3},\left(T_{2,3} ;-3\right)$ is a Seifert fiber space of type $S^{2}(2,3,9)$.

\subsection{The case for hyperbolic knots.}

Throughout this subsection we assume that $K$ is a strongly invertible hyperbolic knot with a cyclic period 2 .

We start with the following observation which shows that $K$ has Type I-C symmetry.

Claim 12.1. We can choose a strong inversion $g$ and a periodic diffeomorphism $f$ realizing the cyclic period 2 of $K$ so that they generate a finite subgroup of Diff* $\left(S^{3}, K\right)$. In particular, $h=g \circ f$ is a strong inversion of $K$.

Proof. Let $f^{\prime}$ be a periodic diffeomorphism giving the cyclic period 2 and $g^{\prime}$ a strong inversion of $K$. Then as in the proof of Corollary 1.2 both $\left[f^{\prime}\right]$ and $\left[g^{\prime}\right]$ are nontrivial and $\left[f^{\prime}\right] \neq\left[g^{\prime}\right]$.

By [5, Theorem 2.1(b)] or [45, Theorem 3.1], we have representing diffeomorphisms $f$ and $g$ so that they generate a finite subgroup of $\operatorname{Diff}^{*}\left(S^{3}, K\right)$ and $[f]=\left[f^{\prime}\right],[g]=\left[g^{\prime}\right]$. Since $g$ reverses an orientation of $K, g$ is also a strong inversion. Moreover from [5, Theorem 2.1 (c)] or more directly from the argument in its proof [5, p.194 line 2-line 5] we see that $f$ gives also the cyclic period of $K$ (see also [52, Corollary 7.2], [45, Theorem 2]). For the last assertion, see the first paragraph of Subsection 6.1. $\square$ (Lemma 12.1)

Then as in Subsection 6.1, we have a finite subgroup $\bar{G} \subset \operatorname{Diff}((K ; r))$ which is generated by two involutions $\bar{f}$ and $\bar{g}$.

Let us show that $(K ; r)$ cannot be a Seifert fiber space of type $S^{2}\left(n_{1}, n_{2}, n_{3}\right)$ with $2<n_{1}<n_{2}<n_{3}$. Assume for a contradiction that $(K ; r)$ is such a Seifert fiber space. Then it has an infinite fundamental group, and hence admits a $\bar{G}$-invariant Seifert fibration by Lemma 3.1 (1).

Since $K$ is assumed to be hyperbolic, [35, Proposition 5.1] shows that $L_{f}^{\prime}$ (the image of $\left.L_{f}=\operatorname{Fix}(f)\right)$ is not a fiber in $(K ; r)$. Then by the argument in the proof of Claim 4.16, $\hat{f}$ is a reflection on $|B|=S^{2}$. Hence by Lemma 8.1 we can assume Condition $(\hat{g})$. If $n_{1}<n_{2}<n_{3}$, then Proposition $5.1(2)$ implies $n_{1}=2$. This contradiction establishes Theorem 1.7 (1) for hyperbolic knots. 
Next we prove that $(K ; r)$ cannot be a Seifert fiber space of type $S^{2}\left(2, n_{2}, n_{3}\right)$ with $\left(n_{2}, n_{3}\right) \neq 1$ and $\left(n_{2}, n_{3}\right) \neq(3,3)$. Again assume to the contrary that $(K ; r)$ has such a Seifert fibration. Since $\left(n_{2}, n_{3}\right) \neq 1$ and $\left(n_{2}, n_{3}\right) \neq(3,3), \frac{1}{2}+\frac{1}{n_{2}}+\frac{1}{n_{3}} \leq 1$ and hence $\left|\pi_{1}((K ; r))\right|=\infty$. Thus as in the above we can assume Condition $(\hat{g})$. Let $\tilde{t_{2}}$ and $\tilde{t_{3}}$ be exceptional fibers of indices $n_{2}$ and $n_{3}$, respectively. Since $n_{2}, n_{3} \geq 3$, by Lemma $5.4 \tilde{t_{2}}$ and $\tilde{t_{3}}$ cover exceptional fibers $t_{2}$ and $t_{3}$ in $(K ; r) /\langle\bar{g}\rangle \cong S^{3}$; since $n_{2} \neq n_{3}$, we have $t_{2} \neq t_{3}$. Note that $\left(\operatorname{index}\left(t_{2}\right)\right.$, index $\left.\left(t_{3}\right)\right)=1$. By Lemma $5.3, n_{i}$ is equal to $\operatorname{index}\left(t_{i}\right), \frac{\operatorname{index}\left(t_{i}\right)}{2}$ or $2 \operatorname{index}\left(t_{i}\right)$. Since $\left(\operatorname{index}\left(t_{2}\right), \operatorname{index}\left(t_{3}\right)\right)=1$ and $\left(2, n_{2}, n_{3}\right)=1,\left(n_{2}, n_{3}\right)=1$ for all possibilities. For instance, assume that $n_{2}=\operatorname{index}\left(t_{2}\right)$ and $n_{3}=2 \operatorname{index}\left(t_{3}\right)$. If $\left(n_{2}, n_{3}\right) \neq 1$, then since $\left(\operatorname{index}\left(t_{2}\right)\right.$, index $\left.\left(t_{3}\right)\right)=1,\left(n_{2}, n_{3}\right)=2$. This is impossible, because $\left(2, n_{2}, n_{3}\right)=1$. This establishes Theorem $1.7(2)$.

\subsection{The case for non-hyperbolic knots.}

Let us assume that $K$ is a strongly invertible satellite knot with a cyclic period 2 and that $(K ; r)$ is a small Seifert fiber space of type $S^{2}\left(n_{1}, n_{2}, n_{3}\right)$ satisfying the condition in Theorem 1.7 (1) or (2). Then by [34, Proposition 2.2] $K$ has a companion solid torus $V$ whose core is a simple knot (i.e., a torus knot or a hyperbolic knot) $k$ in $S^{3}$ and $K(\subset V)$ is a 0 or 1-bridge braid in $V$.

Lemma 12.2. The companion knot $k$ has also a strong inversion and a cyclic periodic 2 .

Proof. Let $\varphi: S^{3} \rightarrow S^{3}$ be a strong inversion of $K$ or an orientation preserving involution which realizes the cyclic period 2 of $K$. Let us choose a $\langle\varphi\rangle$-invariant tubular neighborhood $N(K)$ of $K$. Since the torus decomposition of $S^{3}-\operatorname{int} N(K)$ is unique up to isotopy, we may assume, up to conjugation, that $\varphi$ leaves the companion solid torus $V$ invariant. Note that

$\varphi$ leaves a core $k$ of $V$ invariant. Since $K$ is not homologous to zero in $V, \varphi$ preserves an orientation of $k$ if and only if $\varphi$ preserves an orientation of $K$. It follows that if $\varphi$ is a strong inversion of $K$, then it defines also a strong inversion of $k$, and if $\varphi$ gives a cyclic period 2 of $K$, then it gives also a cyclic period 2 of the companion knot $k$.

$\square$ (Lemma 12.2)

We distinguish two cases depending on whether $K$ is a 0 -bridge braid in the companion solid torus $V$, or $K$ is a 1-bridge braid in $V$. To prove Theorem 1.7 for non-hyperbolic knots, it is sufficient to show that in the first 
case the companion knot $k$ is a torus knot and that the second case cannot happen.

First suppose that $K$ is a 0 -bridge braid in $V$, i.e., $K$ is a $(p, q)$-cable of $k$ for some relatively prime integers $p$ and $q$. Since $(K ; r)=\left(k ; r / q^{2}\right)([20])$ for the simple knot $k$. If $k$ is hyperbolic, then the result in Subsection 12.1, together with Lemma 12.2, gives a contradiction. Thus $k$ is a torus knot as desired.

Remark. If only integral surgery on a hyperbolic knot can yield Seifert fiber space, then no satellite knot with hyperbolic companion can admit a Seifert fibered surgery.

Next we suppose for a contradiction that $K$ is a 1-bridge braid in $V$. Attaching a solid torus $W$ to $V$ so that a preferred longitude of $V$ bounds a meridian disk of $W$, we obtain a new knot $K^{\prime}$ in $S^{3}=V \cup W$ as the image of $K$. Note that since $(K ; r)$ contains no separating incompressible tori, $V(K ; \gamma) \cong S^{1} \times D^{2}$, where $\gamma$ is the slope corresponding to $r$, and hence $\left(K^{\prime} ; \gamma\right)$ is a lens space, possibly $S^{3}$ or $S^{2} \times S^{1}$. On the other hand, Hartley's criterion [26, Theorem 4.1] says that $K^{\prime}$ has also a cyclic period 2 with the axis $J$ a core of $W$. Then from [53], we see that $K^{\prime}$ is a torus knot. Since an involution realizing the cyclic period of $K^{\prime}$ is standard, $K^{\prime}$ is a 0-bridge braid in $V(\subset V \cup W)$. Hence $K$ is also a 0-bridge braid in $V$, contradicting the assumption. This completes the proof of Theorem 1.7 for non-hyperbolic knots.

$\square$ (Theorem 1.7)

\section{References.}

[1] S. A. Bleiler; Prime tangles and composite knots, Lect. Notes in Math. vol. 1144, Springer-Verlag, 1985, pp. 1-13.

[2] S. A. Bleiler and C. D. Hodgson; Spherical space forms and Dehn filling, Topology 35 (1996), 809-833.

[3] M. Boileau and E. Flapan.; Uniqueness of free actions on $S^{3}$ respecting a knot, Can. J. Math. 39 (1987), 969-982.

[4] M. Boileau and J. Porti; Geometrization of 3-orbifolds of cyclic type, (preprint).

[5] M. Boileau and B. Zimmermann; The $\pi$-orbifold group of a link, Math. Z. 200 (1989), 187-208. 
[6] S. Boyer and X. Zhang; Finite Dehn surgery on knots, J. Amer. Math. Soc. 9 (1996), 1005-1050.

[7] S. Boyer and X. Zhang; On Culler-Shalen seminorms and Dehn filling, Ann. Math. 148 (1998), 737-801.

[8] M. Brittenham and Y. Q. Wu; The classification of Dehn surgeries on 2-bridge knots, to appear in Comm. Anal. Geom..

[9] A. Constantin and B. Kolev; The theorem of Kerékjártó on periodic homeomorphisms of the disc and the sphere, L'Enseignement Mathématique 40 (1994), 193-204.

[10] D. Cooper, C. D. Hodgson and S. P. Kerckhoff; Three-dimensional orbifolds and cone-manifolds, MSJ Memoirs, 5, Math. Soc. Japan, 2000.

[11] M. Culler, C. McA. Gordon, J. Luecke, and P. B. Shalen; Dehn surgery on knots, Ann. Math. 125 (1987), 237-300.

[12] J. Dean; Hyperbolic knots with small Seifert-fibered Dehn surgeries, Ph.D. thesis, University of Texas at Austin, 1996.

[13] S. Eilenberg; Sur les transformations périodiques de la surface de la sphère, Fund. Math. 22 (1934), 28-41.

[14] M. Eudave-Muñoz; Non-hyperbolic manifolds obtained by Dehn surgery on a hyperbolic knot, In: Studies in Advanced Mathematics vol. 2, part 1, (ed. W. Kazez), Amer. Math. Soc. and International Press, 1997, pp. 35-61.

[15] M. Eudave-Muñoz; On hyperbolic knots with Seifert fibered Dehn surgeries, (preprint).

[16] D. Gabai; Foliations and the topology of 3-manifolds II, J. Diff. Geom. 26 (1987), 461-478.

[17] D. Gabai; Foliations and the topology of 3-manifolds III, J. Diff. Geom. 26 (1987), 479-536.

[18] D. Gabai; Surgery on knots in solid tori, Topology 28 (1989), 1-6.

[19] F. González-Acuña and H. Short; Knot surgery and primeness, Math. Proc. Camb. Phil. Soc. 99 (1986), 89-102. 
[20] C. McA. Gordon; Dehn surgery and satellite knots, Trans. Amer. Math. Soc. 275 (1983), 687-708.

[21] C. McA. Gordon; Boundary slopes of punctured tori in 3-manifolds, Trans. Amer. Math. Soc. 350 (1998), 1713-1790.

[22] C. McA. Gordon; Dehn Filling; a survey, Proc. Mini Semester in Knot Theory, Banach Center, Warsaw, Poland, 1995.

[23] C. McA. Gordon; Small surfaces and Dehn filling, Proceedings of the Kirbyfest, Geometry and Topology Monographs 2 (1999), 259-290.

[24] C. McA. Gordon and J. Luecke; Dehn surgeries on knots creating essential tori, I, Comm. Anal. Geom. 4 (1995), 597-644.

[25] C. McA. Gordon and J. Luecke; Seifert fibered surgeries on hyperbolic knots, Abstracts Amer. Math. Soc. 20 (1999) and a private communication, November 1998.

[26] R. Hartley; Knots and involutions, Math. Z. 171 (1980), 175-185.

[27] W. Jaco; Lectures on three manifold topology, CBMS Regional Conference Series in Math. 43, Amer. Math. Soc., 1980.

[28] W. Jaco and P. B. Shalen; Seifert fibered spaces in 3-manifolds, Mem. Amer. Math. Soc. 220, 1979.

[29] K. Johannson; Homotopy equivalences of 3-manifolds with boundaries, Lect. Notes in Math. vol. 761, Springer-Verlag, 1979.

[30] J. Luecke; Dehn surgery on knots in the 3-sphere, Proc. International Congress of Mathematicians, Zürich, Switzerland 1994, Birkhaüser Verlag (1995), 585-594.

[31] W. H. Meeks and P. Scott; Finite group actions on 3-manifolds, Invent. math. 86 (1986), 287-346.

[32] W. H. Meeks and S. T. Yau; Equivariant Dehn's lemma and loop theorem, Comment. Math. Helv. 56 (1981), 225-239.

[33] K. Miyazaki and K. Motegi; Seifert fibred manifolds and Dehn surgery, Topology 36 (1997), 579-603.

[34] K. Miyazaki and K. Motegi; Seifert fibered manifolds and Dehn surgery II, Math. Ann. 311 (1998), 647-664. 
[35] K. Miyazaki and K. Motegi; Seifert fibered manifolds and Dehn surgery III, Comm. Anal. Geom. 7 (1999), 551-582.

[36] K. Miyazaki and K. Motegi; Seifert fibering surgery on periodic knots, to appear in Topology Appl.

[37] K. Miyazaki and K. Motegi; On primitive/Seifert-fibered constructions, (preprint).

[38] J. M. Montesinos; Surgery on links and double branched coverings of $S^{3}$, Ann. Math. Stud. 84 (1975), 227-260.

[39] J. W. Morgan and H. Bass (eds.); The Smith conjecture, Academic Press, 1984.

[40] L. Moser; Elementary surgery along a torus knot, Pacific J. Math. 38 (1971), 734-745.

[41] G. Mostow; Quasiconformal mappings in $n$-spaces and the rigidity of hyperbolic space forms, Publ. Math. I.H.E.S. 34 (1968), 53-104.

[42] P. Orlik; Seifert manifolds, Lect. Notes in Math. vol. 291, SpringerVerlag, 1972.

[43] G. Prasad; Strong rigidity of Q-rank 1 lattices, Invent. Math. 21 (1973), $255-283$.

[44] J. H. Rubinstein; On 3-manifolds that have finite fundamental group and contain Klein bottles, Trans. Amer. Math. Soc. 251 (1979), 129137.

[45] M. Sakuma; Realization of the symmetry groups of links, Lect. Notes in Math. vol. 1375, 291-306, Springer-Verlag, 1989.

[46] M. Sakuma; Uniqueness of symmetries of knots, Math. Z. 192 (1986), $225-242$.

[47] P. Scott; The geometries of 3-manifolds, Bull. Lond. Math. Soc. 15 (1983), 401-487.

[48] P. A. Smith; New results and old problems in finite transformation groups, Bull. Amer. Math. Soc. 66 (1960), 401-415.

[49] M. Teragaito; Dehn surgery on genus one knots and Seifert fibered manifolds, Proc. of The 5th Korea-Japan School of Knots and Links, KAIST (1997), 315-325. 
[50] W. P. Thurston; The geometry and topology of 3-manifolds, Lecture notes, Princeton University, 1979.

[51] W. P. Thurston; Three dimensional manifolds, Kleinian groups and hyperbolic geometry, Bull. Amer. Math. Soc. 6 (1982), 357-381.

[52] J. Tollefson; Involutions of sufficiently large 3-manifolds, Topology 20 (1981), 323-352.

[53] S. Wang and Q. Zhou; Symmetry of knots and cyclic surgery, Trans. Amer. Math. Soc. 330 (1992), 665-676.

Department of Mathematics

NiHON UNIVERSITY

Sakurajosui, Setagaya-Ku 3-25-40

TOKYO 156-8550

JAPAN

E-mail: motegi@math.chs.nihon-u.ac.jp

Received June 21, 2001. 